\title{
Modelling in vivo action potential propagation along a giant axon
}

\author{
Stuart George Jamie M. Foster Giles Richardson \\ Received: date / Accepted: date
}

\begin{abstract}
A partial differential equation model for the three-dimensional current flow in an excitable, unmyelinated axon is considered. Where the axon radius is significantly below a critical value $R_{\text {crit }}$ (that depends upon intra- and extra-cellular conductivity and ion channel conductance) the resistance of the intracellular space is significantly higher than that of the extracellular space, such that the potential outside the axon is uniformly small whilst the intracellular potential is approximated by the transmembrane potential. In turn, since the current flow is predominantly axial, it can be shown that the transmembrane potential is approximated by a solution to the one-dimensional cable equation. It is noted that the radius of the squid giant axon, investigated by Hodgkin and Huxley [Hodgkin and Huxley, 1952a], lies close to $R_{\text {crit }}$. This motivates us to apply the three-dimensional model to the squid giant axon and compare the results thus found to those obtained using the cable equation. In the context of the in vitro experiments conducted in [Hodgkin and Huxley, 1952a] we find only a small difference between the wave profiles determined using these two different approaches and little difference between the speeds of action potential propagation predicted. This suggests that the cable equation approximation is accurate in this scenario. However when applied to the in vivo setting, in which the conductivity of the surrounding tissue is considerably lower than that of the axoplasm, there are marked differences in both wave profile and speed of action potential propagation calculated using the two approaches. In particular, the cable equation significantly over predicts the increase in the velocity of propagation
\end{abstract}


as axon radius increases. The consequences of these results are discussed in terms of the evolutionary costs associated with increasing the speed of action potential propagation by increasing axon radius.

\section{Introduction}

Neurons are cells that carry signals throughout organisms, in the form of electric impulses. Many long processes extend from the cell body (which contains the cell's nucleus), divided into two classes. Incoming signals travel through shorter, branching structures known as dendrons and outgoing signals are sent through longer processes called axons [Purves et al., 2011]. Axons are typically long and slender, with an approximately uniform cross-section [Nagarajan and Durand, 1995].

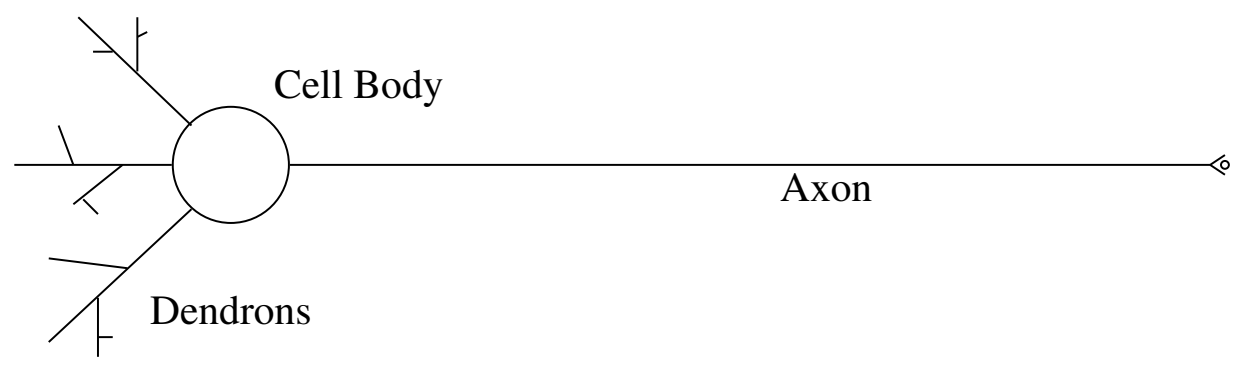

Figure 1: Sketch of neuron. Signals originating in the dendrons are transmitted to the cell body, and then transmitted away along the axon

Integral to the behaviour of a neuron is the composition of its membrane - a phospholipid bilayer which bars the flow of ions between the intracellular and extracellular electrolytes (the intracellular electrolyte is known as axoplasm). The resistance of the membrane to the passage of the different ion species allows for different ion concentrations to be maintained in the axoplasm and the extracellular electrolyte. This is the driving mechanism that leads to a potential jump across the cell membrane (the transmembrane potential).

Ion (and thus current) flow through the membrane is made possible and controlled by 'ion channels' - proteins which span the width of the cell membrane, allowing the passage of a particular ion. Many of these channels are 'voltage-gated', changing their conformation from open to closed 
and vice versa in response to changes in the transmembrane potential [Hille, 2001]. The behaviour of an individual ion channel is stochastic and under certain conditions, this can have significant effects on membrane behaviour, for example causing signals to 'jump' towards clusters of open channels and thus propagate at non-uniform speeds [Faisal and Laughlin, 2007]. However, in larger axons the number of channels is large enough that a homogenised macroscopic model, that considers the average ion channel activity, is appropriate [Strassberg and DeFelice, 1993]. A more detailed discussion of ion channel behaviour and modelling can be found in [Hille, 2001] and the references therein.

Action potentials in squid neurons are primarily driven by flows of potassium and sodium ions. At rest, the cell has relatively high intracellular concentrations of potassium and relatively low intracellular concentrations of sodium, maintained by the action of $\mathrm{Na}^{+} / \mathrm{K}^{+}$-ATPase, an enzyme found in the cell membrane which exchanges intracellular sodium ions for extracellular potassium ions. The cell's resting potential (of around $-70 \mathrm{mV}$ [Purves et al., 2011]) is then predicted by the Goldman equation, which determines the transmembrane potential by balancing the effects of potential gradients with those arising from concentration gradients. The concentration gradients created by $\mathrm{Na}^{+} / \mathrm{K}^{+}$-ATPase provide the energy for the amplification and transmission of neural signalling. Should the cell membrane be depolarised (the transmembrane potential raised) above a threshold by some local injection of current, a cycle of responses occur which lead to a propagating signal. The time constant (that is, the characteristic time scale over which the channel responds to transmembrane potential changes) for the opening of sodium channels $\left.\left(\sim 10^{-4} \mathrm{~s}\right)\right)$ is much smaller than that of the potassium $\left.\left(\sim 5 \times 10^{-3} \mathrm{~s}\right)\right)$ [Gerstner and Kistler, 2002, Hodgkin and Huxley, 1952a], which means that the initial current flow in response to the depolarisation is almost entirely due to the movement of sodium ions. Sodium channels open in response to the depolarisation and the concentration and potential gradients cause positively charged sodium ions to flow into the cell from the extracellular medium, further depolarising it and beginning a positive feedback loop which opens further sodium channels. This sodium current is responsible for the sharp upward spike seen on the trace of an action potential (such traces are common in any discussion of the basics of action potential mechanics, see for example [Purves et al., 2011]). As the transmembrane potential increases, the potassium channels also begin to open, and the potassium concentration gradient causes positively charged potassium ions to flow out of the cell. On 
a similar timescale the sodium channels inactivate, preventing further flow of positive ions into the cell, and so the transmembrane potential returns to its resting value (after a slight 'overshoot', known as a hyperpolarisation). As the local membrane depolarisation in response to the influx of sodium ions reaches its peak $(\sim 40 \mathrm{mV})$ it subsequently raises the membrane potential in neighbouring regions above the threshold required to begin the action potential cycle. Thus, as each region of membrane reaches its peak potential and excites the regions adjacent to it, the action potential propagates along the axon in the form of a travelling wave.

In this paper we are primarily concerned with the behaviour of the squid giant axon, an unusually large axon (up to around $1 \mathrm{~mm}$ diameter) found in the mantle of some species of squid, although large axons in general are found in other types of invertebrate (e.g. aplysia [Hughes and Tauc, 1963] and lobster [Bean, 1981]). These giant axons mediate the squid's 'escape response', which allows it to rapidly evade potential threats such as predators [Preuss and Gilly, 2000, Otis and Gilly, 1990]. Larger axons are known to transmit action potentials faster, and since the speed of the escape response is critical to the survival of the squid this accounts for the large size of the giant axon. Increasing the size of the axon comes with an increased cost to the squid, in terms of the energy needed for the growth, maintenance and operation of the cell, and thus we expect some kind of 'optimal' size of squid giant axon, balancing these factors.

The 'voltage-gated' behaviour of the ion channels was the subject of the seminal work of Hodgkin and Huxley, in a series of papers published in 1952 [Hodgkin and Huxley, 1952b,c,d,e,a, Hodgkin et al., 1952]. The work described in these papers was centred around empirical measurements of the conductance of the membrane of the squid giant axon to different ions at different membrane potentials. While this model was not based on the microscopic electrophysiology of ion channels (their existence was only conclusively demonstrated in the 1970s [Katz and Miledi, 1973], and they were not directly observed until the invention of the patch clamp [Neher and Sakmann, 1976, Neher et al., 1978]), it nevertheless shows how the different conductances in the axon membrane interact to produce the action potential.

In addition to a description of membrane currents, Hodgkin and Huxley (and many others since) used the cable equation to describe the longitudinal propagation of action potentials along axons [Hodgkin and Huxley, 1952a]. The cable equation is a nonlinear diffusion equation, originally used to study signal transmission in transatlantic telegraph cables [Thompson, 1855] but 
quickly adapted for use in neurobiology. Key to the use of this model are the implicit assumptions that current flow within the axon is predominantly axial and that the potentials outside the axon are uniformly small (this is equivalent to requiring that the resistance of the axon to be significantly larger than that of the extracellular space). This approach yields good agreement with empirical studies - Hodgkin and Huxley tested their model by taking parameters from a particular axon, measuring the speed of signal transmission within this axon, and then comparing the result with a travelling wave solution to their model. The empirical speed was found to be $21.2 \mathrm{~ms}^{-1}$, which compares favourably to the $18.8 \mathrm{~ms}^{-1}$ predicted by their model [Hodgkin and Huxley, 1952a]. The source of this error has motivated a number of subsequent works. In particular, improvements have been made to ion channel conductance models that take advantage of the wealth of information about ion channel structure now available, improved empirical data on single-channel behaviour, and knowledge of the features of excitable membranes, such as thresholds and refractory periods [Perram and Stiles, 2010, Vandenberg and Benzanilla, 1991]. These have been found to significantly reduce the disagreement between theory and experiment [Armstrong and Benzanilla, 1977, Benzanilla and Armstrong, 1977].

As discussed in section 2 and appendix A, a key requirement for the cable equation to be an appropriate approximation to axonal behaviour is that the axon is sufficiently thin (equivalent to it having a sufficiently large ratio of intracellular to extracellular resistance). This condition is satisfied for a wide range of axons, however some - and in particular the squid giant axons used by Hodgkin and Huxley — inhabit a grey area where it is not obvious that this criterion is met.

An important feature of the Hodgkin-Huxley experiment [Hodgkin and Huxley, 1952a], and indeed most other experiments on squid axons since ([Adair, 2004],[Rattay, 1986]) is that it is conducted in vitro with an excised axon suspended in a bath of seawater, an electrolyte with relatively high conductivity. The external conditions in a live squid are rather different as, in vivo, the axon is surrounded by a collagenous sheath, as well as other closely packed cells, all acting to decrease the conductivity of the extracellular space relative to the intracellular space [Bear et al., 1937]. This, as we shall demonstrate, has important consequences for the validity of the cable equation approximation in vivo, and means that it breaks down at significantly smaller radii than in the highly conductive external medium of seawater used by Hodgkin and Huxley. A corollary of the breakdown of the cable equation 
approximation is that action potential propagation velocities do not scale with the square root of axon radius, but instead saturate to some constant value (for large radii). Based on our estimate of extracellular conductivity we suggest that physiological axon radius is close to optimal (certainly further gains in action potential propagation speed become increasingly expensive).

A useful observation on signalling speed can be made by examining travelling wave solutions to the cable equation (as noted by Hodgkin [1954]). We begin with the cable equation (as used by Hodgkin and Huxley, and derived from the underlying electrochemical equations in appendix A)

$$
C \frac{\partial \Phi^{*}}{\partial t^{*}}=\frac{\sigma R}{2} \frac{\partial^{2} \Phi^{*}}{\partial x^{* 2}}-j^{*}\left(\Phi^{*}\left(x^{*}, t^{*}\right), \vec{w}\left(x^{*}, t^{*}\right)\right),
$$

where $\Phi^{*}$ is the transmembrane potential, $j^{*}$ represents the current density through the ion channels, $x^{*}$ is distance along the axon, $t^{*}$ is time, $C$ is the capacitance of the membrane per unit area, $\sigma$ is the conductivity of the axoplasm and $R$ is the axon radius (a $*$ denotes a dimensional variable). Specifically, the Hodgkin-Huxley form of the membrane current $j^{*}$ has the form

$$
\begin{gathered}
j^{*}\left(\Phi^{*}, \vec{w}\right)=\sum_{i=1}^{N} g_{i}^{*} w_{i}\left(\Phi^{*}-\Phi_{e i}\right), \\
\frac{\partial w_{i}}{\partial t}=\alpha_{i}^{*}\left(\Phi^{*}\right)\left(1-w_{i}\right)-\beta_{i}^{*}\left(\Phi^{*}\right) w_{i}
\end{gathered}
$$

where $\Phi_{e i}$ is the reversal potential for each ion species, $g_{i}^{*}$ is the maximal membrane conductance per unit area of the $i$ th ion species, $w_{i}$ is the proportion of this conductance accessible through open ion channels and $\alpha_{i}^{*}$ and $\beta_{i}^{*}$ are functions (which are given in Hodgkin and Huxley [1952a]) that describe opening and closing rates for each species of ion channel.

Making the travelling wave ansatz $\Phi^{*}=f(\xi)$ and $w_{i}=\omega_{i}(\xi)$, where $\xi=\frac{x^{*}}{v}-t^{*}$ gives

$$
\begin{aligned}
-C \frac{d f}{d \xi} & =\frac{\sigma R}{2 v^{2}} \frac{d^{2} f}{d \xi^{2}}-\sum_{i} \omega_{i}(\xi)\left(f(\xi)-\Phi_{e i}\right), \\
\frac{d w_{i}}{d \xi} & =-\left(\alpha_{i}^{*}(f(\xi))\left(1-w_{i}(\xi)\right)-\beta_{i}^{*}(f(\xi)) w_{i}(\xi)\right),
\end{aligned}
$$

and imposing the boundary conditions $f \rightarrow 0$ as $\xi \rightarrow \pm \infty$, so that the membrane is at resting potential far from the propagating wave, yields a 
nonlinear eigenvalue problem, with eigenvalue $\frac{\sigma R}{2 v^{2}}$. This gives us the following result for the wave speed, $v$ (assuming membrane capacitance to be fixed)

$$
v \propto \sigma^{\frac{1}{2}} R^{\frac{1}{2}}
$$

such that the propagation velocity of the signal in the axon scales with the square root of the axon radius. We expect our model to reproduce this behaviour for sufficiently small radii (since the cable equation is valid in the asymptotic limit as axon radius goes to zero), and larger extracellular to intracellular conductance ratios, but for larger radii and smaller ratios we expect to observe differing predictions from the two models. Later in this study we will revisit this notion of travelling wave speeds and make a comparison between predictions from our model and that of Hodgkin and Huxley. By doing this we can both assess the range of parameters over which their approximation is justified and gain an understanding of the behaviour of very large axons, in configurations for which the cable equation is unsuitable.

\section{Model Formulation}

Here we formulate a generic model of the current flow in the vicinity of an axon whilst noting that this model reduces to the cable equation in the limit of small axon thickness (as described in Richardson and SJ [2011]).

This model of current flow has been previously used to describe both neurons [Lindsay et al., 2004, Richardson, 2009] and other electrochemically excitable cells, such as cardiac myocytes [Krassowska and Neu, 1993, Richardson and SJ, 2011]. Furthermore it has been systematically derived from a detailed asymptotic analysis of the Nernst-Planck equation of electrochemistry [Richardson, 2009].

With the relatively small currents encountered during action potentials, the electrolyte behaviour in the interior and exterior regions of the axon (denoted by $\Omega^{*}$ and $\Omega^{c *}$ respectively) is well approximated by Ohm's law and current conservation

$$
\begin{array}{cccc}
\vec{J}^{*}=-\sigma_{\text {in }} \nabla^{*} \phi^{*} & \text { and } & \nabla^{*} \cdot \vec{J}^{*}=0 & \text { in } \Omega^{*}, \\
\vec{J}^{*}=-\sigma_{\text {out }} \nabla^{*} \phi^{*} & \text { and } & \nabla^{*} \cdot \vec{J}^{*}=0 & \text { in } \Omega^{c *}
\end{array}
$$

where $\overrightarrow{J^{*}}$ and $\phi^{*}$ denote current density and electric potential respectively, and $\sigma_{\text {in,out }}$ represents the conductivity of the electrolyte in the interior and 
exterior regions. It follows that the potential satisfies Laplace's equation in both the interior and exterior regions

$$
\nabla^{* 2} \phi^{*}=0 \text { in } \Omega^{*} \text { and } \Omega^{c *} .
$$

We also require a far-field condition on $\phi^{*}$ as distance from the axon membrane tends to infinity. (The precise statement of this condition is dependent on the geometry of the axon under consideration, and thus we do not define it rigorously until the axon geometry is fixed).

The behaviour of the membrane is modelled with reference to the ion channels in the membrane, the membrane permittivity and the behaviour of the extremely narrow $(\sim 1 \mathrm{~nm})$ charged Debye layers lying on either side of it. It is possible to demonstrate that the charge densities lying in the Debye layers on either side of the membrane are equal and opposite ([Richardson and SJ, 2011]) such that the membrane and Debye layers behave as a capacitor. As a corollary the macroscopic current density flowing across membrane and Debye layers is continuous

$$
\left.\overrightarrow{J^{*}} \cdot \vec{n}^{*}\right|_{\partial \Omega^{*}}=\left.\vec{J}^{*} \cdot \vec{n}^{*}\right|_{\partial \Omega^{c *}},
$$

where $\vec{n}^{*}$ the unit vector normal to the membrane. For sufficiently small transmembrane potentials, such as those encountered in an action potential, the membrane (and its Debye layers) behave as a linear capacitor ([Richardson, 2009]) whilst the ion channels straddling it can be modelled as a nonlinear resistor with current-voltage dependency given by $j^{*}\left(\Phi^{*}, t^{*}\right)$ in parallel with this capacitor. The equivalent circuit is shown in figure 2 and the cor-

responding transmembrane current density $\left.\vec{J}^{*} \cdot \vec{n}^{*}\right|_{\partial \Omega^{*}}$ (where this includes the Debye layers) satisfies

$$
\left.\vec{J}^{*} \cdot \vec{n}^{*}\right|_{\partial \Omega^{*}}=C \frac{\partial \Phi^{*}}{\partial t^{*}}+j^{*}\left(\Phi^{*}, t^{*}\right)
$$

\subsection{Model closure}

Equations (7)-(10), with a suitable far-field condition, now comprise the following axon-scale model for the electrolyte potential and the transmembrane 


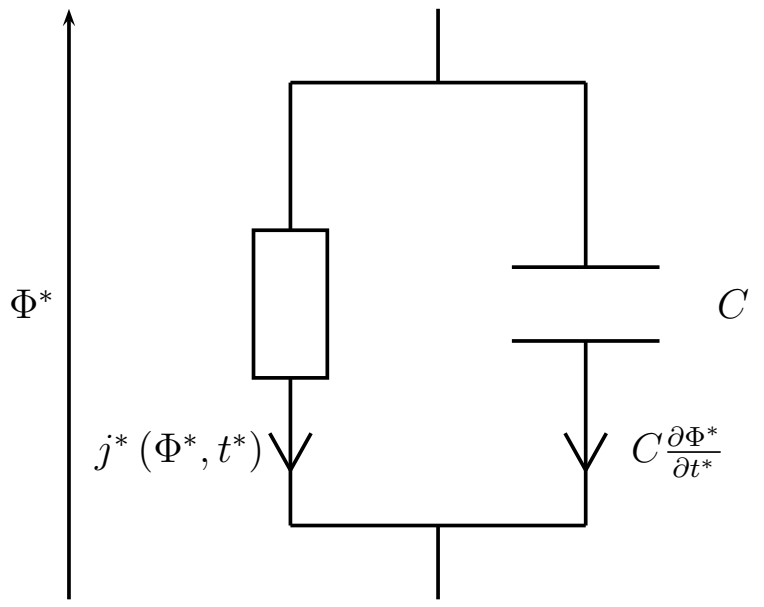

Figure 2: Equivalent circuit diagram for currents through the cell membrane

potential, $\Phi^{*}$, defined as the difference between the intra- and extra-cellular potentials.

$$
\begin{aligned}
\nabla^{* 2} \phi^{*} & =0 \text { in } \Omega \text { and } \Omega^{c}, \\
\left.\sigma_{\text {in }} \frac{\partial \phi^{*}}{\partial n^{*}}\right|_{\partial \Omega^{*}} & =\left.\sigma_{\text {out }} \frac{\partial \phi^{*}}{\partial n^{*}}\right|_{\partial \Omega^{c *}}, \\
{\left[\phi^{*}\right]_{\partial \Omega^{c}}^{\partial \Omega} } & =\Phi, \\
C \frac{\partial \Phi^{*}}{\partial t^{*}} & =-\left.\sigma_{\text {in }} \frac{\partial \phi^{*}}{\partial n^{*}}\right|_{\partial \Omega^{*}}-j^{*} .
\end{aligned}
$$

The exact form of the equations used to describe the current through the ion channels, $j^{*}$, is dependent on the kind of membrane being modelled. For squid axons the seminal model is, as mentioned above, that of Hodgkin and Huxley. This model describes three separate current densities, two active currents caused by the flow of potassium and sodium ions through specific ion channels and a generalised non-specific Ohmic 'leak' current. For each ion species there exists some maximal current density (obtained when all ion channels, through which that species can travel, are open), and the actual current density is then a proportion of this maximum dependent on how many channels are in the open state.

Specifically, the Hodgkin-Huxley form of the membrane current is given 
by

$$
\begin{aligned}
j^{*} & =\sum_{i=1}^{N} g_{i}^{*} w_{i}\left(\Phi^{*}-\Phi_{e i}\right), \\
\frac{\partial w_{i}}{\partial t^{*}} & =\alpha_{i}^{*}\left(\Phi^{*}\right)\left(1-w_{i}\right)-\beta_{i}^{*}\left(\Phi^{*}\right) w_{i},
\end{aligned}
$$

with $g_{i}^{*}, w_{i}$ and $\Phi_{e i}$ as described in section 1 [Hodgkin and Huxley, 1952a].

\subsubsection{Nondimensionalisation}

We nondimensionalise via the scalings

$$
\begin{aligned}
& \vec{x}^{*}=L \vec{x}, \quad t^{*}=\tau t, \quad \Phi^{*}=\Phi_{0} \Phi, \quad \phi^{*}=\Phi_{0} \phi, \\
& g_{i}^{*}=g_{0} g, \quad j^{*}=g_{0} \Phi_{0} j, \quad \alpha^{*}=\frac{1}{\tau} \alpha, \quad \beta^{*}=\frac{1}{\tau} \beta .
\end{aligned}
$$

Here $\tau$ represents the typical timescale for an action potential, $\Phi_{0}$ a typical transmembrane potential and $g_{0}$ a typical membrane conductance per unit area. We choose the length scale $L$ by balancing the axoplasm conductivity per unit length with membrane conductance per unit area

$$
L=\frac{\sigma_{\mathrm{in}}}{g_{0}}
$$

such that equations (12)-(17) can be written in the form

$$
\begin{aligned}
\nabla^{2} \phi & =0 \text { in } \Omega \text { and } \Omega^{c} \\
\left.\frac{\partial \phi}{\partial n}\right|_{\partial \Omega} & =\left.\bar{\sigma} \frac{\partial \phi}{\partial n}\right|_{\partial \Omega^{c}}, \\
{[\phi]_{\partial \Omega^{c}}^{\partial \Omega} } & =\Phi \\
\mathcal{C} \frac{\partial \Phi}{\partial t} & =-\left.\frac{\partial \phi}{\partial n}\right|_{\partial \Omega}-j
\end{aligned}
$$

where

$$
\begin{aligned}
j & =\sum_{i=1}^{N} g_{i} w_{i}\left(\Phi-\Phi_{e i}\right) \\
\frac{\partial w_{i}}{\partial t} & =\alpha_{i}(\Phi)\left(1-w_{i}\right)-\beta_{i}(\Phi) w_{i}
\end{aligned}
$$


The dimensionless parameters $\mathcal{C}$ and $\bar{\sigma}$ are defined by

$$
\mathcal{C}=\frac{C}{\tau g_{0}} \quad \text { and } \quad \bar{\sigma}=\frac{\sigma_{\text {out }}}{\sigma_{\text {in }}},
$$

and represent the ratio of charge stored in the Debye layers to charge moved through the ion channels in a typical action potential and the ratio of extracellular to intracellular conductivities respectively. We choose $\Phi_{0}$ to be the thermal voltage $\left(\sim 2.5 \times 10^{-2} \mathrm{~V}\right)$, which is comparable to a typical transmembrane potential (at rest, transmembrane potential $\sim-7 \times 10^{-2} \mathrm{~V}$ ) and the typical membrane conductance $g_{0}$ to be the maximal conductance through the voltage-gated sodium channels $\left(\sim 1200 \mathrm{~S} \cdot \mathrm{m}^{-2}\right.$ [Llano et al., 1988]). Values for the parameters $C$ and $\sigma_{\text {in }}$ can be found in the literature $\left(C \sim 1 \times 10^{-2} \mathrm{~F} \cdot \mathrm{m}^{-2}\right.$ [Hodgkin and Huxley, 1952a] and $\sigma_{\text {in }} \sim 1 \mathrm{~S} \cdot \mathrm{m}^{-1}$ [Stampfli, 1952] $\left(=2.825 \mathrm{~S} \cdot \mathrm{m}^{-1}\right.$ in Hodgkin and Huxley [1952a])), while the effect of several values of $\sigma_{\text {out }}$ is discussed in section 4 .

In the rest of this work we will be mainly concerned with uniform cylindrical axons such that another important dimensionless parameter is the ratio of the axon radius, $R^{*}$, to the lengthscale, $L$, which we denote by $\epsilon=R / L$. Where this parameter is very small the resistance of the interior of the axon is large compared to that of the extracellular space so that extracellular po-

tentials are small while the potential inside the axon is well approximated by the local transmembrane potential, $\Phi$ (see appendix A and [Richardson, 2009]). Using the parameter values chosen above, $L=O(2.5 \mathrm{~mm})$, yielding $\epsilon \approx 0.2$ for larger squid axons $(\epsilon=0.10$ for the exact parameters in Hodgkin and Huxley [1952a]). It is not obvious (as discussed above) whether this is small enough for the cable equation to yield a good approximation.

In the case of a cylindrical axon, for $\epsilon \ll 1$ and $\bar{\sigma}=1$, equations (20)-(23) can be simplified to

$$
\mathcal{C} \frac{\partial \Phi}{\partial t}=\frac{\epsilon}{2} \frac{\partial^{2} \Phi}{\partial x^{2}}-j(\Phi, t)
$$

as derived in appendix A.

\section{Method}

In the following sections we formulate a numerical procedure for solving equations (20)-(23) without approximating it by the cable equation or exploiting 
the smallness of $\epsilon$. We then apply this procedure specifically to a uniform cylindrical axon.

Initially we approach the problem by introducing a Green's function, $G\left(\vec{x} ; \overrightarrow{x_{0}}\right)$, defined as the solution to

$$
\begin{aligned}
\nabla^{2} G & =0 \\
\left.\frac{\partial G}{\partial n}\right|_{\partial \Omega} & =\left.\bar{\sigma} \frac{\partial G}{\partial n}\right|_{\partial \Omega^{c}} \\
{[G]_{\partial \Omega^{c}}^{\partial \Omega} } & =\delta\left(\vec{x}-\overrightarrow{x_{0}}\right)
\end{aligned}
$$

again with a suitable far-field condition (in terms of the Green's function, $G \rightarrow 0$ as distance from the axon membrane becomes large), where $\overrightarrow{x_{0}}$ denotes a point on the axon membrane, and $\delta(\cdot)$ the Dirac delta function.

The solution to equations $(20)-(22)$ can then be written in terms of a surface integral over the axon surface $\partial \Omega$

$$
\phi(\vec{x}, t)=f_{\partial \Omega_{\vec{x}_{0}}} G\left(\vec{x}, \vec{x}_{0}\right) \Phi\left(\vec{x}_{0}, t\right) d \Omega_{\vec{x}_{0}}
$$

such that equation (23) gives an integral equation for $\Phi$

$$
\mathcal{C} \frac{\partial}{\partial t} \Phi(\vec{x}, t)=-\left.\frac{\partial}{\partial n}\left(\int_{\partial \Omega_{\vec{x}_{0}}} G\left(\vec{x}, \vec{x}_{0}\right) \Phi\left(\vec{x}_{0}, t\right) d \Omega_{\vec{x}_{0}}\right)\right|_{\partial \Omega}-j
$$

in which $j$ is given by equations $(24)-(25)$.

\subsection{A uniform cylindrical axon}

We now consider the specific case of a uniform cylindrical axon of dimensionless radius $\epsilon=\frac{R^{*}}{L}$, as seen in figure 3, with axisymmetric membrane potential $\Phi$. We rewrite equation $(31)$ in cylindrical polar coordinates $(x, y, z)=$ $(x, r \cos (\theta), r \sin (\theta))$ as follows:

$$
\phi(x, r, \theta, t)=f_{-\infty}^{\infty} \int_{0}^{2 \pi} G\left(x, r, \theta ; x_{0}, \theta_{0}\right) \epsilon d \theta_{0} \Phi\left(x_{0}, t\right) d x_{0} .
$$

With the assumption that $\Phi$ is axisymmetric we can integrate over $\theta$, i.e.

$$
\mathcal{G}\left(x-x_{0}, r\right)=\int_{0}^{2 \pi} G\left(x, r, \theta ; x_{0}, \theta_{0}\right) d \theta_{0}
$$




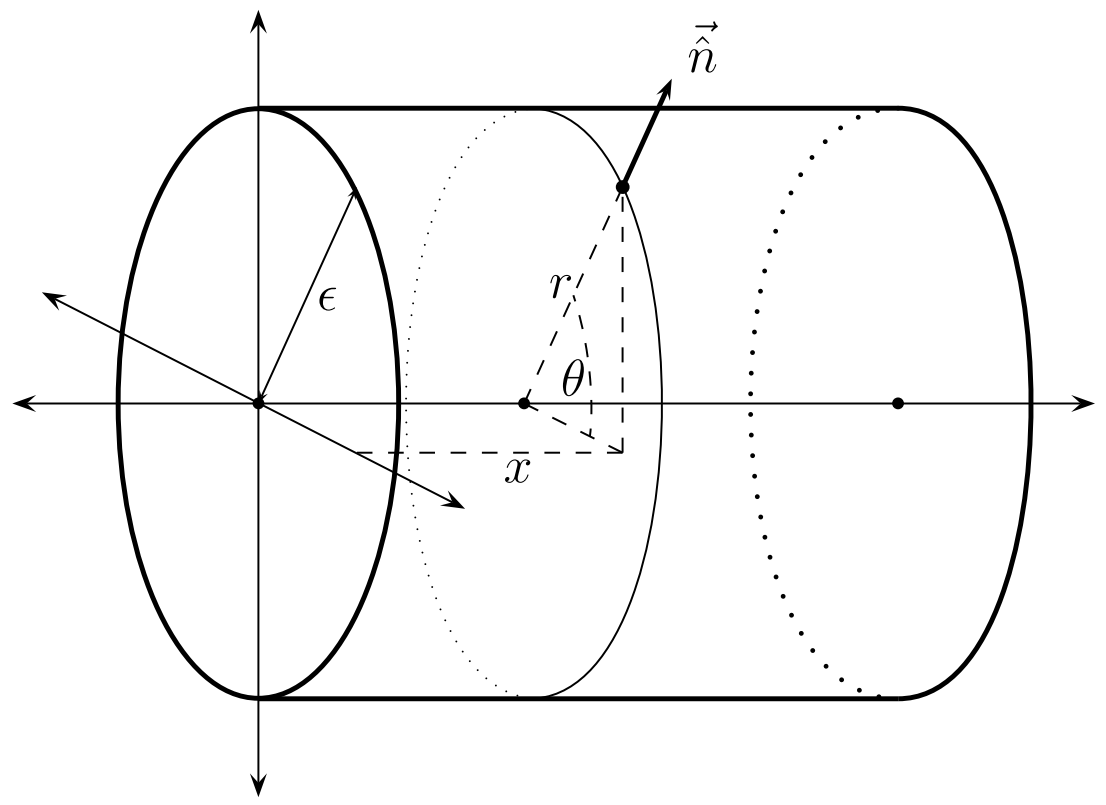

Figure 3: Schematic of the cylindrical geometry. The unit normal vector $\overrightarrow{\hat{n}}$ is directed out of the cylinder

so that $\mathcal{G}$ is the Green's function for a 'ring' of charge around the axon membrane, located at $x_{0}$. We observe that the axial dependence of $G$ occurs solely as a function of $x-x_{0}$ and that integration of equations (28)-(30) over $\theta$ (on noting that $\delta\left(\vec{x}-\overrightarrow{x_{0}}\right)=\frac{1}{\epsilon} \delta\left(x-x_{0}\right) \delta\left(\theta-\theta_{0}\right)$ ) yields the following problem for $\mathcal{G}(X, r)$ :

$$
\begin{gathered}
\frac{\partial^{2} \mathcal{G}}{\partial X^{2}}+\frac{1}{r} \frac{\partial}{\partial r}\left(r \frac{\partial \mathcal{G}}{\partial r}\right)=0 \text { in } r<\epsilon \text { and } r>\epsilon, \\
\left.\frac{\partial \mathcal{G}}{\partial r}\right|_{r=\epsilon^{-}}=\left.\bar{\sigma} \frac{\partial \mathcal{G}}{\partial r}\right|_{r=\epsilon^{+}}, \\
{[\mathcal{G}]_{r=\epsilon^{+}}^{r=\epsilon^{-}}=\frac{1}{\epsilon} \delta(X),} \\
\mathcal{G} \rightarrow 0 \text { as } r \rightarrow \infty .
\end{gathered}
$$

Equation (32) can be rewritten in terms of this axisymmetric Green's function, $\mathcal{G}$ in the form

$$
\mathcal{C} \frac{\partial \Phi}{\partial t}=-\left.\frac{\partial}{\partial r}\left(f_{-\infty}^{\infty} \mathcal{G}\left(x-x_{0}, r\right) \Phi\left(x_{0}, t\right) \epsilon d x_{0}\right)\right|_{r=\epsilon^{-}}-j(x, t),
$$


where $j$ is given by equations (24)-(25).

Ideally, we would like to be able to replicate the approach in section 1 to find the relationship between signal speed and axon radius in this model. Making the change of variables $\xi=t-\frac{x}{v}$ results in the nonlinear integrodifferential eigenvalue problem

$$
\begin{gathered}
\mathcal{C} \frac{d}{d \xi} \Phi(\xi)=-\left.\frac{\partial}{\partial r}\left(f_{-\infty}^{\infty} \mathcal{G}\left(v\left(\xi-\xi_{0}\right), r\right) \Phi\left(\xi_{0}\right) \epsilon v d \xi_{0}\right)\right|_{r=\epsilon^{-}}-\sum_{i=1}^{N} g_{i} w_{i}(\xi)\left(\Phi(\xi)-\Phi_{e i}\right) \\
\frac{d w_{i}}{d \xi}=-\left(\alpha_{i}(\Phi(\xi))\left(1-w_{i}(\xi)\right)-\beta_{i}(\Phi(\xi)) w_{i}(\xi)\right) \\
\Phi(\xi) \rightarrow 0 \quad \text { as } \xi \rightarrow \pm \infty
\end{gathered}
$$

The eigenvalue $v$ again depends upon $\epsilon$. In section 1 we were able to explicitly determine how wave speed scales with $\epsilon$, but the nature of the integral operator in this problem makes direct solution of this nonlinear eigenvalue problem non-trivial. Instead, we solve the time-dependent problem numerically and allow the solution to converge to the travelling wave and measure

the speed of the subsequent wave. This allows us to calculate the eigenvalue (for all $\mathcal{C}$ ) by performing one computation for each value of $\epsilon$.

\subsection{Numerical Method}

We briefly outline the key points of our numerical scheme below and relegate the exact details to appendix B for brevity. Here we shall assume that $\Phi$ is periodic with some large period $2 \lambda$ - so that in the limit $\lambda \rightarrow \infty$ we retrieve the exact result. We adapt the Green's function in equations (35)-(38) to reflect the periodic nature of the problem

$$
\begin{gathered}
\frac{\partial^{2} \mathcal{G}}{\partial X^{2}}+\frac{1}{r} \frac{\partial}{\partial r}\left(r \frac{\partial \mathcal{G}}{\partial r}\right)=0 \quad \text { in } r<\epsilon \text { and } r>\epsilon, \\
\left.\frac{\partial \mathcal{G}}{\partial r}\right|_{r=\epsilon^{-}}=\left.\bar{\sigma} \frac{\partial \mathcal{G}}{\partial r}\right|_{r=\epsilon^{+}}, \\
{[\mathcal{G}]_{r=\epsilon^{+}}^{r=\epsilon^{-}}=\frac{1}{\epsilon} \sum_{n=-\infty}^{\infty} \delta(X+2 n \lambda),} \\
\mathcal{G} \rightarrow 0 \quad \text { as } \quad r \rightarrow \infty
\end{gathered}
$$


We solve equations (43)-(46) using the method of Fourier series to find the following expression for $\mathcal{G}$

$$
\mathcal{G}(X, r)=\left\{\begin{array}{ll}
\frac{1}{\lambda}+\sum_{n=1}^{\infty} \frac{\bar{\sigma} \mathcal{K}_{1}\left(\frac{n \pi \epsilon}{\lambda}\right)}{\lambda\left(\bar{\sigma} \mathcal{I}_{0}\left(\frac{n \pi \epsilon}{\lambda}\right) \mathcal{K}_{1}\left(\frac{n \pi \epsilon}{\lambda}\right)+\mathcal{I}_{1}\left(\frac{n \pi \epsilon}{\lambda}\right) \mathcal{K}_{0}\left(\frac{n \pi \epsilon}{\lambda}\right)\right)} \mathcal{I}_{0}\left(\frac{n \pi r}{\lambda}\right) \cos \left(\frac{n \pi X}{\lambda}\right) & \text { for } r<\epsilon \\
-\sum_{n=1}^{\infty} \frac{\mathcal{I}_{1}\left(\frac{n \pi \epsilon}{\lambda}\right)}{\lambda\left(\bar{\sigma} \mathcal{I}_{0}\left(\frac{n \pi \epsilon}{\lambda}\right) \mathcal{K}_{1}\left(\frac{n \pi \epsilon}{\lambda}\right)+\mathcal{I}_{1}\left(\frac{n \pi \epsilon}{\lambda}\right) \mathcal{K}_{0}\left(\frac{n \pi \epsilon}{\lambda}\right)\right)} \mathcal{K}_{0}\left(\frac{n \pi r}{\lambda}\right) \cos \left(\frac{n \pi X}{\lambda}\right) & \text { for } r>\epsilon
\end{array},\right.
$$

where $\mathcal{I}_{0}(\cdot)$ and $\mathcal{I}_{1}(\cdot)$ are modified Bessel functions of the first kind and $\mathcal{K}_{0}(\cdot)$ and $\mathcal{K}_{1}(\cdot)$ are modified Bessel functions of the second kind. More details of the derivation of this solution are given in appendix B.

We note that the singular integral term in equation (39) is a Fourier convolution of $\left.\frac{\partial \mathcal{G}}{\partial r}\right|_{r=\epsilon^{-}}$and $\Phi$. This suggests that the equation may be tractable to a spectral method and leads us to consider the Fourier series of $\Phi, \mathcal{G}$ and $j$ which we write in the form

$$
\begin{gathered}
\Phi(x, t)=P_{0}(t)+\sum_{n=1}^{\infty} P_{n}(t) \cos \left(\frac{n \pi x}{\lambda}\right), \\
\mathcal{G}(x, r)=g_{0}(r)+\sum_{n=1}^{\infty} g_{n}(r) \cos \left(\frac{n \pi x}{\lambda}\right), \\
j(x, t)=j_{0}(t)+\sum_{n=1}^{\infty} j_{n}(t) \cos \left(\frac{n \pi x}{\lambda}\right) .
\end{gathered}
$$

On noting that the $n$ 'th term in the Fourier cosine series of the convolution $(f \star g)(x)$ is $\frac{1}{2} f_{n} g_{n}$ (where $f_{n}$ and $g_{n}$ are the $n$ 'th terms in the Fourier cosine series of the functions $f(x)$ and $g(x)$, respectively) we note that equation (39) can be transformed to

$$
\frac{d P_{n}}{d t}=-\left.\frac{\lambda}{2 \mathcal{C}} \frac{d g_{n}}{d r}\right|_{r=\epsilon^{-}} P_{n}-j_{n} .
$$

Given a functional dependence for $j(x, t)$ this formulation of the problem allows us to use a Runge-Kutta method to solve for $P_{n}(t)$ and thus for $\Phi(x, t)$ and has the notable advantage, over solving equation (39) directly, that the singularity in $\mathcal{G}$ can be dealt with easily. In the frequency domain, this singularity occurs in the limit $n \rightarrow \infty$, as the Bessel functions in equation (47) also tend to infinity. We note that due to equation (51), large and 
positive $g_{n}$ simply implies that $P_{n} \rightarrow 0$ very quickly, or equivalently that very high frequency components of our solution decay very quickly.

Numerical solution of equation (51) via a spectral method. Given an initial condition $\Phi(x, 0)$, we calculate initial conditions for the gating variables $w_{i}$ by the assumption that the axon is at rest, and thus $w_{i}(x, 0)$ is the steady state solution to equation (25), and we use these values to determine the membrane current $j(x, 0)$ through equation $(24)$. Once $\Phi$ and $j$ are known, we make use of the fast Fourier transform (FFT) to determine the coefficients $P_{n}$ and $j_{n}$ in equation (51). We can also use the values of $\Phi$ and $w_{i}$ (in the time domain) to find the derivatives in equation (25). This allows us to write the time derivatives of $P_{n}$ and $w_{i}$ in the form

$$
\begin{aligned}
& \frac{d P_{n}}{d t}=f_{1}\left(t, x, P_{n}, j_{n}\right) \\
& \frac{d w_{i}}{d t}=f_{2, i}\left(t, x, \Phi, w_{i}\right)
\end{aligned}
$$

which is amenable to solution using a standard, fourth order, Runge-Kutta method. The only complication here is that we have to use an inverse fast Fourier transform at each iteration, turning the updated values $P_{n}$ into an updated $\Phi$, calculating new gating variable values and then new membrane channel values in the time domain, and finally using the FFT to convert back into the frequency domain. A sensible choice of $\lambda$ depends on the choices we make for other parameters in our model (namely $\epsilon$ and $\sigma_{\text {in }}$, and the length of time for which we run the simulation) but we note that the results presented in this paper are robust for $\lambda>750$.

In appendix $\mathrm{C}$ we derive an analytical solution to a simplified version of this problem (by replacing the Hodgkin Huxley ion channel dynamics with a linear membrane resistance) and note the favourable comparison between this solution and the results of our simulations.

\section{Results}

We use our algorithm to calculate solutions to our model for a range of dimensionless axon radii $\epsilon$, and for a range of conductivities. The results of these simulations are compared to solutions of the equivalent cable equation in order to gauge its validity both in vitro and in vivo. 
The case in vitro $(\bar{\sigma}=1)$. We begin by looking at the case where intracellular and extracellular conductivities are equal which we believe to be the case in the in vitro experiments conducted in Hodgkin and Huxley [1952a]. We note the divergence of our results from those predicted by the cable equation as $\epsilon$ increases, as expected. However, as shown in figure 4, this divergence does not become significant until the dimensionless axon radius is considerably larger than that typically occurring in a squid. A comparison between our solutions and those of the cable equation is also informative, and we note that the cable equation slightly overestimates the width of the wave profile (see figure 5). Figure 6 shows cross-sections of the intracellular and extracellular potentials generated during an action potential. The magnitude of the extracellular potentials generated in the smaller axon $(\epsilon=0.1$, top) are less than a third those of the intracellular potentials, resulting in the close agreement between the cable equation predictions and the results of our simulations. The size of the extracellular potentials increases slightly as $\epsilon$ increases, but not enough (over the physiological range of radii) to mean that the cable equation approach ceases to give an accurate estimate of action potential velocity.

The case in vivo $(\bar{\sigma}=0.1)$. An estimate for the extracellular conductivity of a nerve bundle of $\sigma_{\text {out }}=0.385 \mathrm{~S} \cdot \mathrm{m}^{-1}$ is given in Altman and Plonsey [1990], which is roughly one tenth of the intracellular conductivity used by Hodgkin and Huxley (and therefore yields $\bar{\sigma}=0.1$ in our model). We must note that the determination of these parameters is non-trivial, and significant variation exists in measurements of both intracellular and extracellular conductivities. We feel, however, that this only strengthens the argument for considering scenarios where $\bar{\sigma} \neq 1$.

For the case, where $\bar{\sigma}=0.1$,we find that the divergence from the solutions of the cable equation (in terms of propagation speed, in figure 7 , and travelling wave profile, in figure 8) is much more pronounced, and becomes apparent for a physiologically viable range of axon radii, suggesting that the cable equation is an inaccurate model for the behaviour of axons in vivo. The cross-sections in figure 9 explain this inaccuracy - the extracellular potential adjacent to the axon has become comparable to the intracellular potential adjacent to the axon, violating the assumptions required to derive the cable 


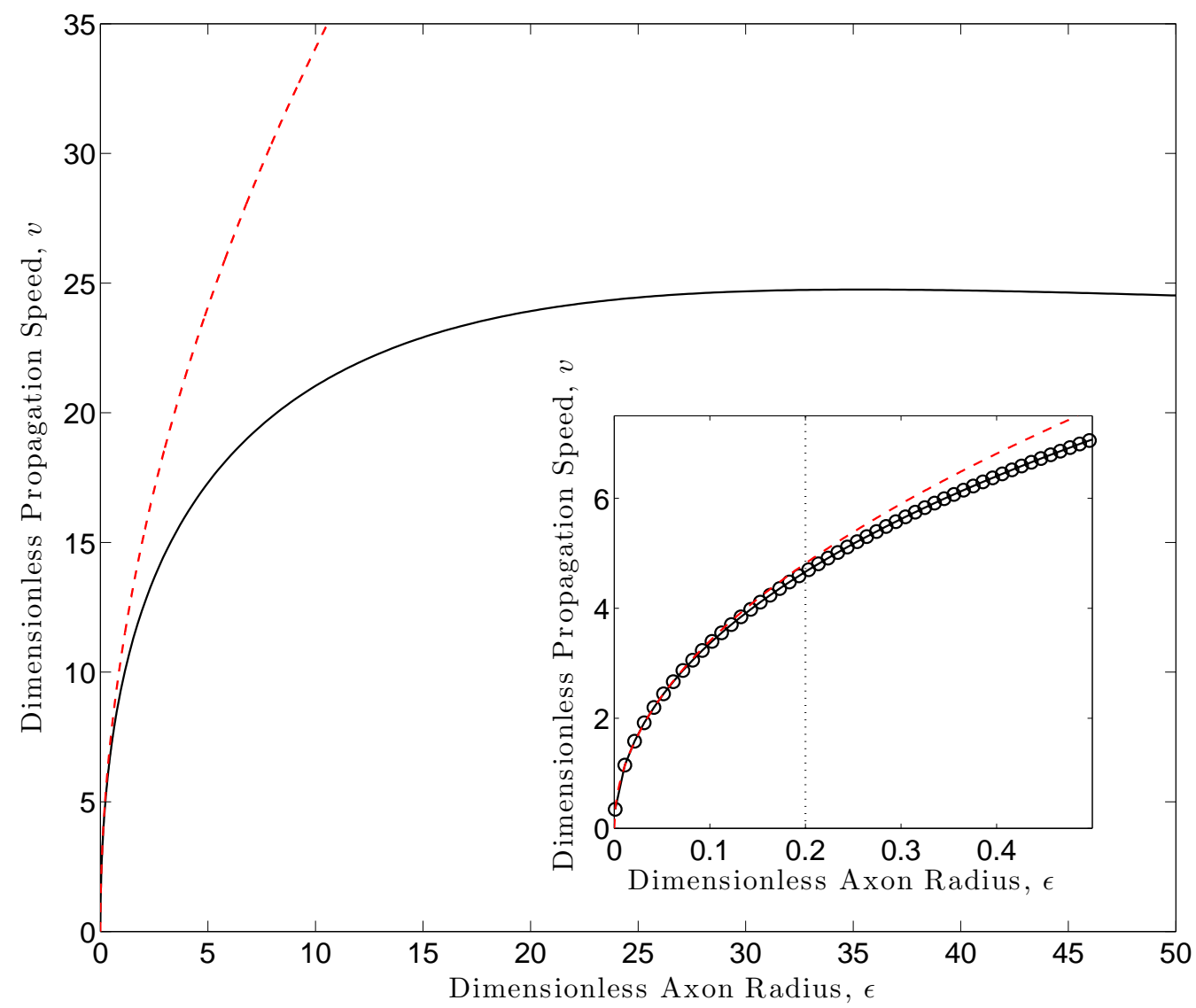

Figure 4: Variation of dimensionless propagation speed, $v$, with dimensionless axon radius, $\epsilon$, with $\bar{\sigma}=1$, as calculated by our simulation (solid, black curve) and predicted by the cable equation (dashed, red curve). Dotted line on inset is at $\epsilon=0.2$, the approximate upper bound on $\epsilon$, corresponding to the largest squid axons. Over a physiological range of $\epsilon$, the discrepancy between the two models is not significant. Simulations were run with $\lambda=750$, for $t=0$ to 30 with a time step of 0.001 and 8192 space steps 


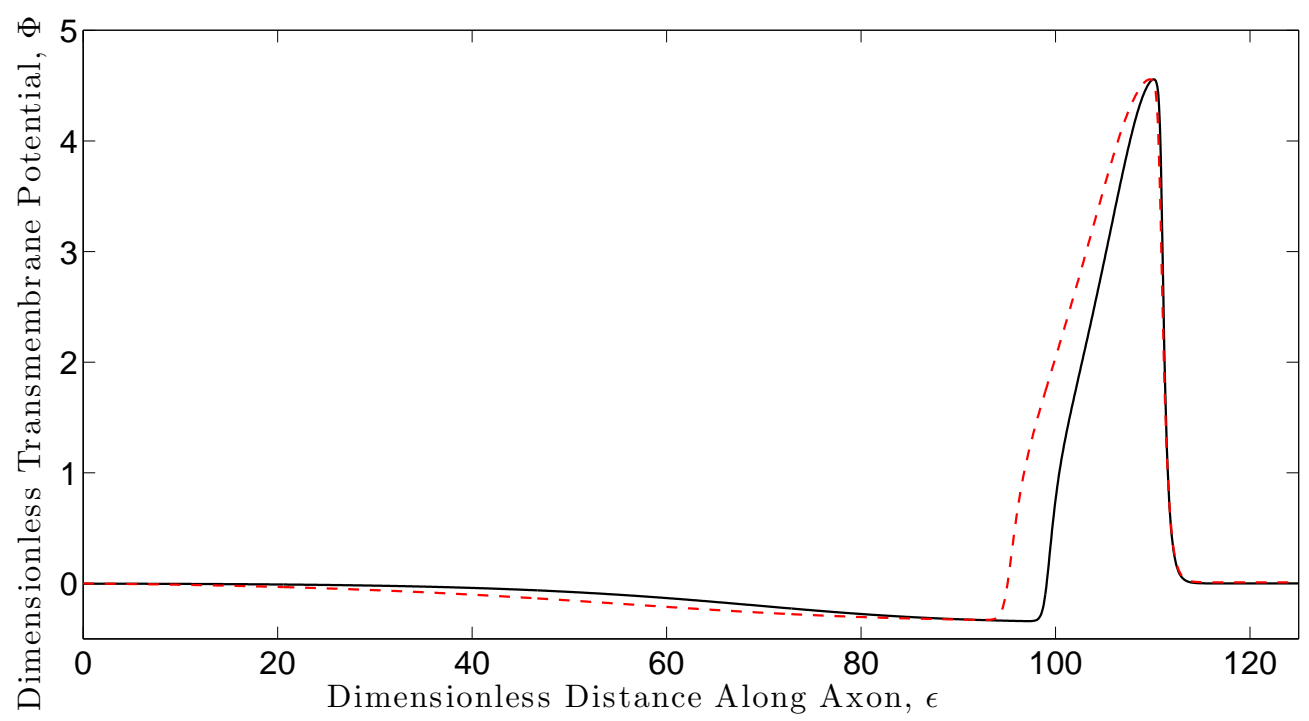

Figure 5: A typical travelling wave profile from our simulation (solid, black curve) and the cable equation (dashed, red curve) for the in vitro case, $\epsilon=$ $0.2, \bar{\sigma}=1$

equation.

Figure 10 shows contour plots of the potentials generated close to the axon membrane during the propagation of an action potential. As mentioned above, the extracellular potentials are seen to be much larger in the case $\bar{\sigma}=0.1$ (left-hand column), and as such the cable equation is a much worse approximation to this case.

\section{Discussion}

At least one of a squid's escape responses is initiated by an action potential propagating along its giant axon, which triggers the contraction of the circular muscle groups around its siphon and gives rise to a powerful propulsive jet of water [Preuss and Gilly, 2000, Otis and Gilly, 1990]. Fast action potential propagation (along the giant axon) is thus associated with rapid 


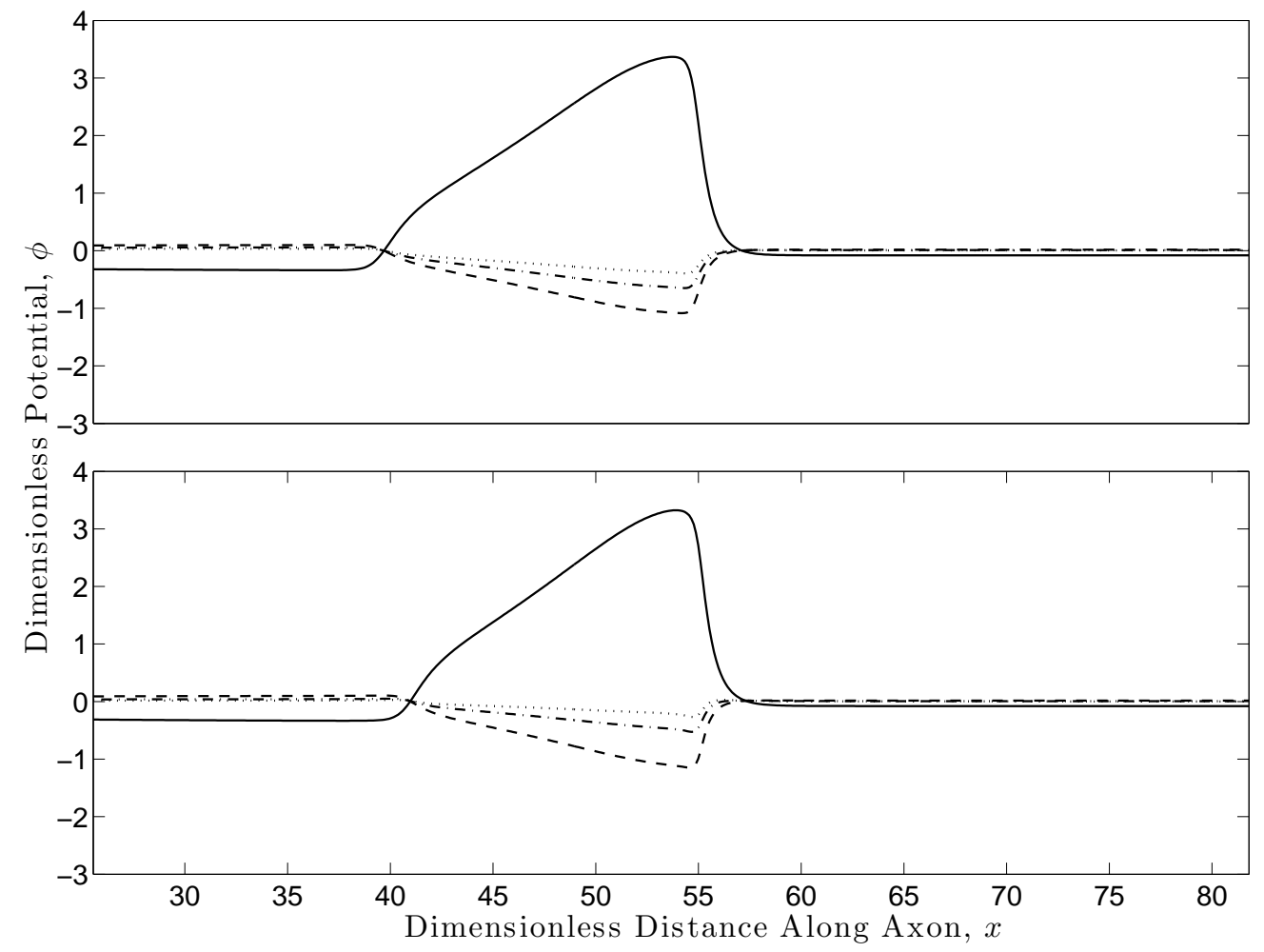

Figure 6: Cross-section of intracellular (solid curve) and extracellular potentials during an action potential, in axons of dimensionless radius $\epsilon=0.1$ (upper panel) and $\epsilon=0.3$ (lower panel), for $\bar{\sigma}=1$. Intracellular cross-section is taken at $r=\epsilon-10^{-6}$, with extracellular cross-sections at $r=\epsilon+10^{-6}$ (dashed curve), $r=1.05 \epsilon$ (dashed and dotted curve) and $r=1.1 \epsilon$ (dotted curve) 


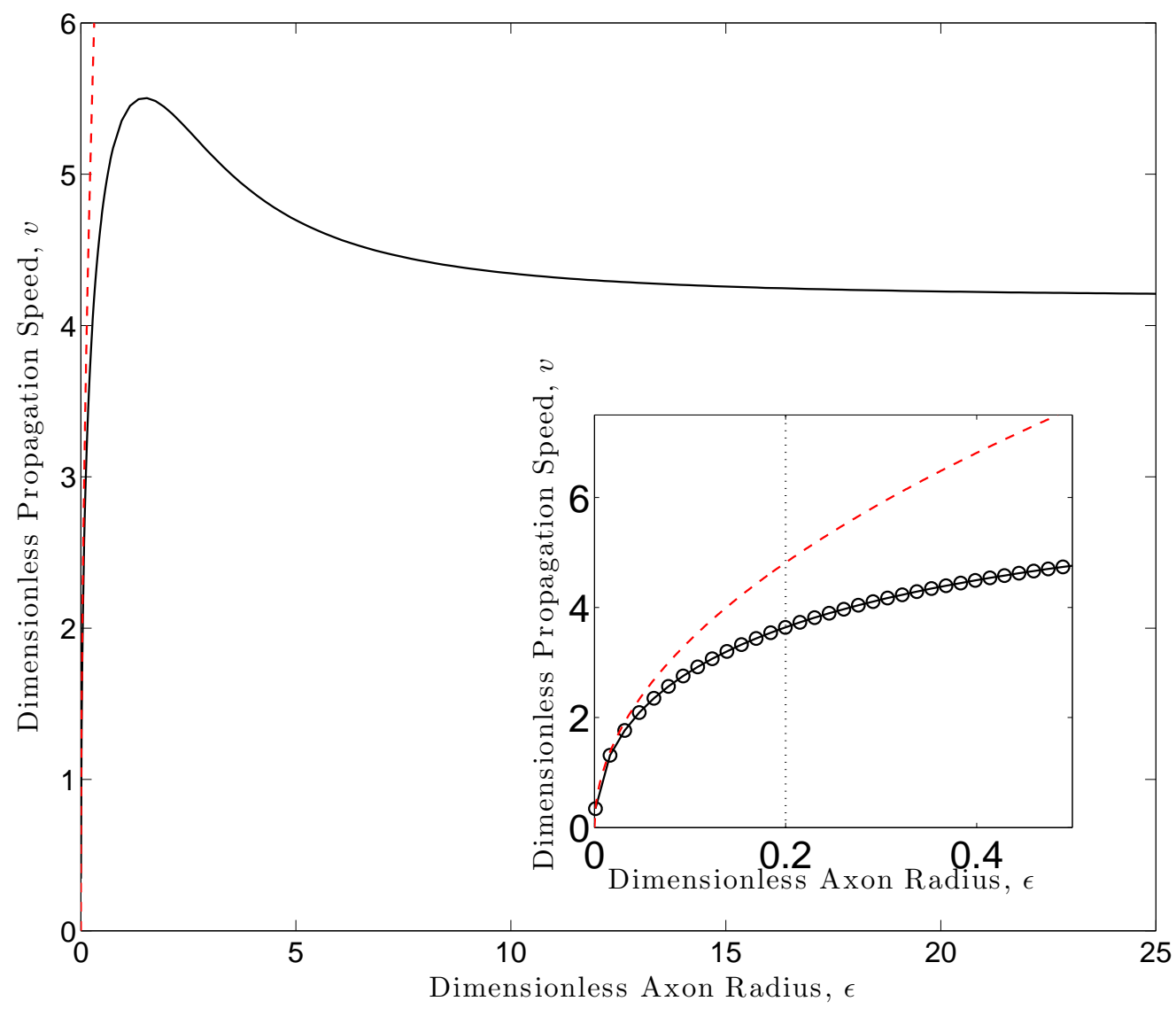

Figure 7: Variation of dimensionless propagation speed, $v$, with dimensionless axon radius, $\epsilon$, with $\bar{\sigma}=0.1$, as calculated by our simulation (solid, black curve) and predicted by the cable equation (dashed, red curve). Dotted line on inset is at $\epsilon=0.2$, the approximate upper bound on $\epsilon$, corresponding to the largest squid axons. The discrepancy between the two models over a physiological range of $\epsilon$ is clear. Simulation parameters are identical to the case $\bar{\sigma}=1$ 


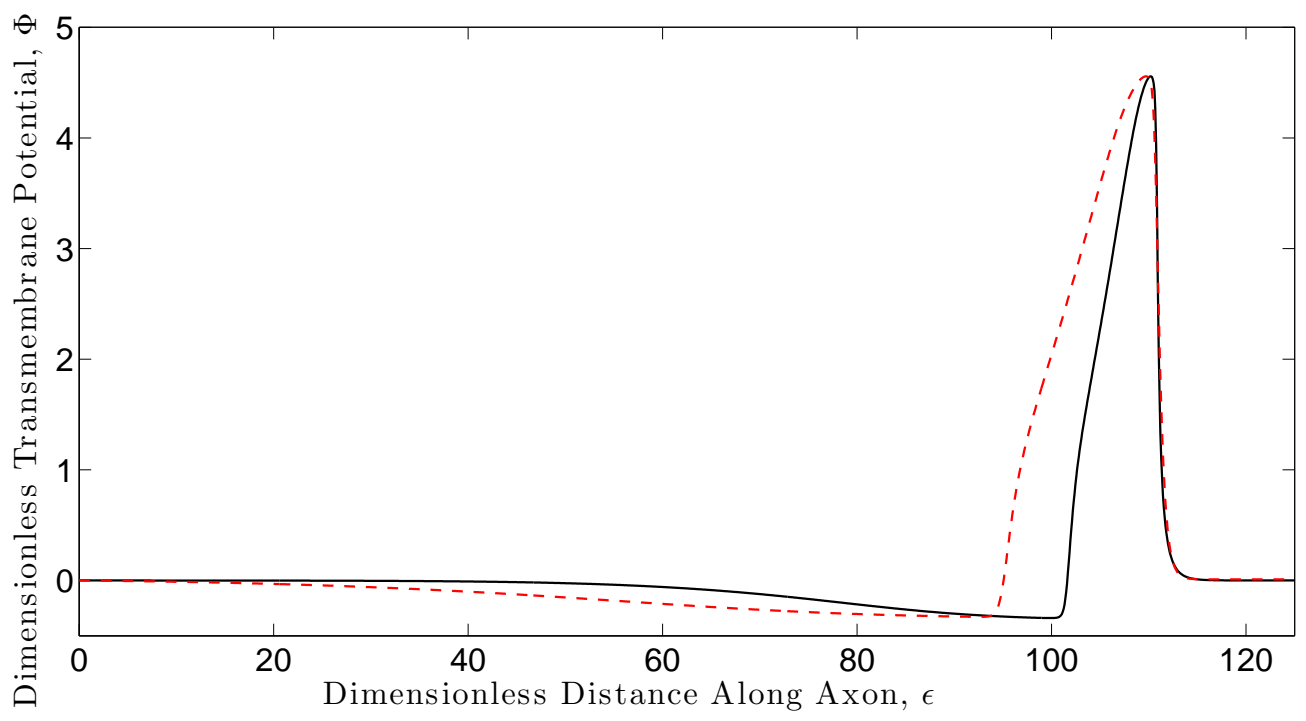

Figure 8: A typical travelling wave profile from our simulation (solid, black curve) and the cable equation (dashed, red curve) for the in vivo case, $\epsilon=$ $0.2, \bar{\sigma}=0.1$

escape and is therefore expected to be a characteristic that is under strong selection pressure.

Study of the cable equation model of action potential propagation predicts that signal conduction speed is maximised by maximising the axoplasmic conductivity of the axon or the axon radius. The fact that the cable equation predicts that propagation speed will scale like $R^{\frac{1}{2}}$ without limit means that it does not predict an 'optimal' axon size, simply that bigger is better. Instead, arguments concerning the increased metabolic cost of growing and maintaining a larger axon are used to explain why the squid giant axon is not larger than it is.

Our approach suggests that there is a law of diminishing returns (in terms of increased propagation velocity for increases in axon radius) which means - in the physiological case for a large axon $(\bar{\sigma}=0.1, R \approx 0.5 \mathrm{~mm})$ that increases in propagation velocity scale significantly more slowly with increases in axon radius, $R$, than the $R^{\frac{1}{2}}$ dependence predicted by the cable equation. In addition there is a maximal radius, for a given conductivity ratio $\bar{\sigma}$, at which the propagation velocity obtains a maximum value and above which further increases in axon size result in a decrease in propagation speed. 


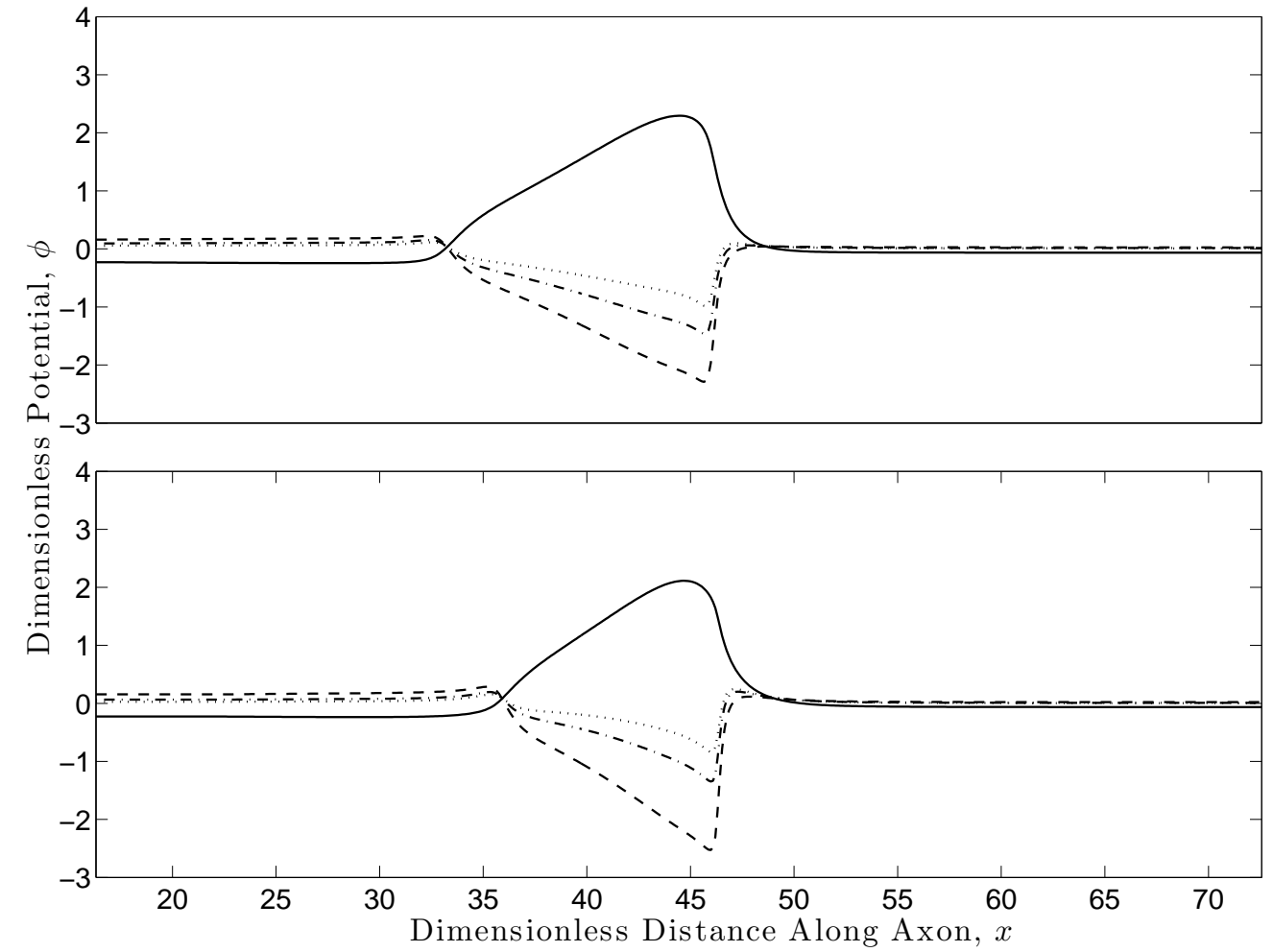

Figure 9: Cross-section of intracellular (solid curve) and extracellular potentials during an action potential, in axons of dimensionless radius $\epsilon=0.1$ (upper panel) and $\epsilon=0.3$ (lower panel), for $\bar{\sigma}=0.1$. Intracellular cross-section is taken at $r=\epsilon-10^{-6}$, with extracellular cross-sections at $r=\epsilon+10^{-6}$ (dashed curve), $r=1.05 \epsilon$ (dashed and dotted curve) and $r=1.1 \epsilon$ (dotted curve). 


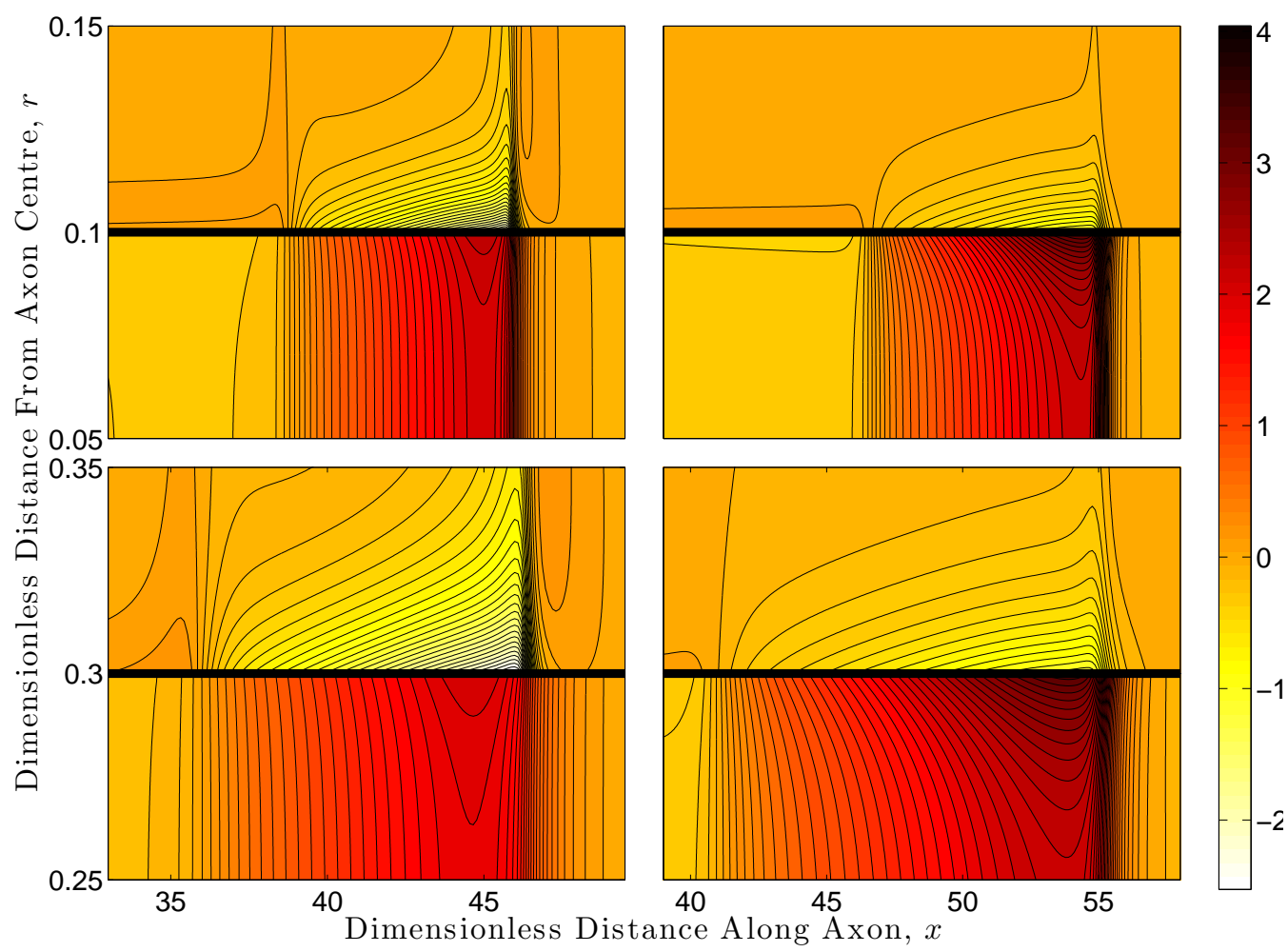

Figure 10: Contour plots of intracellular and extracellular potentials generated by the propagation of an action potential, for dimensionless radii $\epsilon=0.1$ (upper panels) and $\epsilon=0.3$ (lower panels) and $\bar{\sigma}=0.1$ (left panels) and $\bar{\sigma}=1$ (right panels). The thick solid line through the centre of each panel shows the location of the axon membrane 
We note however that even in the physiological case $\bar{\sigma}=0.1$ the optimal radius is significantly larger than any physiologically feasible giant axon. This suggests that metabolic cost is still a limiting factor in determining the size of the axon, and that diminishing returns (in terms of increased propagation velocity) on increased metabolic investment in axon size are not sufficient to justify further increases in size.

\section{A Deriving the cable equation approximation}

We consider a uniform cylindrical axon with dimensionless radius $\epsilon \ll 1$. In this case, equations (20)-(23) and the far-field condition can be written as [Richardson, 2009]

$$
\begin{aligned}
\nabla^{2} \phi & =0 \text { in } r<\epsilon \text { and } r>\epsilon \\
\left.\frac{\partial \phi}{\partial r}\right|_{r=\epsilon^{-}} & =\left.\bar{\sigma} \frac{\partial \phi}{\partial r}\right|_{r=\epsilon^{+}}, \\
{[\phi]_{r=\epsilon^{+}}^{r=\epsilon^{-}} } & =\Phi, \\
\mathcal{C} \frac{\partial \Phi}{\partial t} & =-\left.\frac{\partial \phi}{\partial r}\right|_{r=\epsilon^{-}}-j(\Phi, t) .
\end{aligned}
$$

We solve this problem by introducing inner and exterior regions, distances $O(\epsilon)$ and $O\left(\epsilon^{\frac{1}{2}}\right)$ from the axon, respectively. As we will demonstrate, the cable equation can be derived from the equations in the inner region alone. A detailed treatment of the solution for the outer region is given in Richardson [2009]. We distinguish inner variables with superscript (in). In this region we rescale coordinates as follows:

$$
x=\epsilon^{\frac{1}{2}} \xi \quad r=\epsilon \rho,
$$


which leads us to the following system:

$$
\begin{aligned}
& \frac{1}{\epsilon \rho} \frac{\partial}{\partial \rho}\left(\rho \frac{\partial \phi^{(\mathrm{in})}}{\partial \rho}\right)+\frac{\partial^{2} \phi^{(\mathrm{in})}}{\partial \xi^{2}}=0 \\
& {\left[\phi^{(\mathrm{in})}\right]_{\rho=1^{+}}^{\rho=1^{-}}=\Phi,} \\
& \left.\frac{\partial \phi^{(\mathrm{in})}}{\partial \rho}\right|_{\rho=1^{-}}=\left.\bar{\sigma} \frac{\partial \phi^{(\mathrm{in})}}{\partial \rho}\right|_{\rho=1^{+}}, \\
& \mathcal{C} \frac{\partial \Phi}{\partial t}=-\left.\frac{1}{\epsilon} \frac{\partial \phi^{(\mathrm{in})}}{\partial \rho}\right|_{\rho=1^{-}}-j .
\end{aligned}
$$

We look for a solution of the following form (where we have included $O(\epsilon \log (\epsilon))$ terms due to the far-field logarithmic singularity encountered when solving Laplace's equation in cylindrical coordinates). The assumption that $\phi^{(\mathrm{in})}$ in $\rho>1$ is small being made here $\left(\phi^{(\text {in })}=O(\epsilon \log \epsilon)\right)$ is key to the understanding of why this simplification breaks down where it does. As noted in section 4 this is not true once the axon radius becomes sufficiently large such that the intracellular resistance is comparable to the effective extracellular resistance. Furthermore this critical radius diminishes as the extracellular conductivity decreases (since decreases in extracellular conductivity increase extracellular resistance).

$$
\begin{aligned}
\Phi & =\Phi_{0}+\epsilon \log (\epsilon) \Phi_{1}+\epsilon \Phi_{2}+\cdots, \\
\phi^{(\mathrm{in})} & =\left\{\begin{array}{cl}
\Phi_{0}+\epsilon \log (\epsilon)\left(\phi_{1}^{(\mathrm{in})}+\Phi_{1}\right)+\epsilon\left(\phi_{2}^{(\mathrm{in})}+\Phi_{2}\right)+\cdots & \text { for } 0 \leq \rho<1 \\
\epsilon \log (\epsilon) \phi_{1}^{(\mathrm{in})}+\epsilon \phi_{2}^{(\mathrm{in})}+\cdots & \text { for } \rho>1
\end{array}\right.
\end{aligned}
$$

Substitution of this expansion into equations (59)-(62) gives (at $O(1)$ )

$$
\begin{array}{rlrl}
\frac{1}{\rho} \frac{\partial}{\partial \rho}\left(\rho \frac{\partial \phi_{2}^{(\mathrm{in})}}{\partial \rho}\right) & =-\Phi_{0, \xi \xi} & \rho<1 \\
\frac{1}{\rho} \frac{\partial}{\partial \rho}\left(\rho \frac{\partial \phi_{2}^{(\mathrm{in})}}{\partial \rho}\right) & =0 \quad \rho>1, \\
{\left[\phi_{2}^{(\mathrm{in})}\right]_{\rho=1^{+}}^{\rho=1^{-}}} & =0, \\
\left.\frac{\partial \phi_{2}^{(\mathrm{in})}}{\partial \rho}\right|_{\rho=1^{-}} & =\left.\bar{\sigma} \frac{\partial \phi_{2}^{(\mathrm{in})}}{\partial \rho}\right|_{\rho=1^{+}},
\end{array}
$$


which has the solution

$$
\phi_{2}^{(\mathrm{in})}=\left\{\begin{array}{ll}
-\frac{1}{4} \Phi_{0, \xi \xi}\left(\rho^{2}-1\right)+\gamma(\xi, t) & \text { for } \rho<1 \\
-\frac{1}{2 \bar{\sigma}} \Phi_{0, \xi \xi} \log (\rho)+\gamma(\xi, t) & \text { for } \rho>1
\end{array} .\right.
$$

Substitution of equation (69) into equation (62) yields the following version of the cable equation

$$
\mathcal{C} \frac{\partial \Phi_{0}}{\partial t}=\frac{1}{2} \frac{\partial^{2} \Phi_{0}}{\partial \xi^{2}}-j .
$$

\section{B Calculation of the Green's function $\mathcal{G}$}

Here we look for a solution to equations (43)-(46) for the Green's function $\mathcal{G}(X, r)$ in terms of its Fourier series

$$
\mathcal{G}(X, r)=\frac{g_{0}}{2}+\sum_{n=1}^{\infty} g_{n} \cos \left(\frac{n \pi X}{\lambda}\right)
$$

Substitution of equation (71) into equation (43) yields

$$
\begin{aligned}
\frac{\partial^{2} \mathcal{G}}{\partial X^{2}}+\frac{1}{r} \frac{\partial}{\partial r}\left(r \frac{\partial \mathcal{G}}{\partial r}\right)= & \sum_{n=1}^{\infty} g_{n}^{\prime \prime} \cos \left(\frac{n \pi X}{\lambda}\right)+\frac{1}{r} \sum_{n=1}^{\infty} g_{n}^{\prime} \cos \left(\frac{n \pi X}{\lambda}\right) \\
& -\frac{\pi^{2}}{\lambda^{2}} \sum_{n=1}^{\infty} n^{2} g_{n} \cos \left(\frac{n \pi X}{\lambda}\right)+\frac{g_{0}^{\prime \prime}}{2}+\frac{1}{2 r} g_{0}^{\prime}=0 \quad \text { in } r<\epsilon \text { and } r>\epsilon,
\end{aligned}
$$

which leads to the following ODEs for the functions $g_{n}(r)$

$$
\begin{aligned}
g_{0}^{\prime \prime}+\frac{1}{r} g_{0}^{\prime} & =0 \\
g_{n}^{\prime \prime}+\frac{g_{n}^{\prime}}{r}-\left(\frac{n \pi}{\lambda}\right)^{2} g_{n} & =0 \quad \text { for } \quad n \geq 1 .
\end{aligned}
$$

Solution of equation (73) yields

$$
g_{0}= \begin{cases}c_{1,0}+c_{2,0} \ln (r) & \text { for } r<\epsilon \\ d_{1,0}+d_{2,0} \ln (r) & \text { for } r>\epsilon\end{cases}
$$


In order to avoid a singular solution at $r=0$ and as $r \rightarrow \infty$, we require $c_{2,0}=d_{2,0}=0$. Solution of equation (74) gives

$$
g_{n}=\left\{\begin{array}{ll}
c_{1, n} \mathcal{I}_{0}\left(\frac{n \pi r}{\lambda}\right)+c_{2, n} \mathcal{K}_{0}\left(\frac{n \pi r}{\lambda}\right) & \text { for } r<\epsilon \\
d_{1, n} \mathcal{I}_{0}\left(\frac{n \pi r}{\lambda}\right)+d_{2, n} \mathcal{K}_{0}\left(\frac{n \pi r}{\lambda}\right) & \text { for } r>\epsilon
\end{array} .\right.
$$

Again we require $c_{2, n}=d_{1, n}=0$, to avoid singularities in this solution, leaving

$$
g_{n}=\left\{\begin{array}{ll}
c_{1, n} \mathcal{I}_{0}\left(\frac{n \pi r}{\lambda}\right) & \text { for } r<\epsilon \\
d_{1, n} \mathcal{K}_{0}\left(\frac{n \pi r}{\lambda}\right) & \text { for } r>\epsilon
\end{array} .\right.
$$

The jump condition on $\mathcal{G}$ (equation (45)) is used to fix $c_{1, n}$ in terms of $d_{1, n}$ for all $n$ as follows:

$$
\begin{aligned}
\delta\left(x-x_{0}\right) & =\frac{1}{\lambda}+\sum_{n=1}^{\infty} \frac{1}{\lambda} \cos \left(\frac{n \pi\left(x-x_{0}\right)}{\lambda}\right), \\
{\left[g_{0}\right]_{r=\epsilon^{+}}^{r=\epsilon^{-}} } & =c_{1,0}-d_{1,0}=\frac{1}{\lambda}, \\
{\left[g_{n}\right]_{r=\epsilon^{+}}^{r=\epsilon^{-}} } & =\left(c_{1, n} \mathcal{I}_{0}\left(\frac{n \pi r}{\lambda}\right)-d_{1, n} \mathcal{K}_{0}\left(\frac{n \pi r}{\lambda}\right)\right)=\frac{1}{\lambda}, \\
& \Rightarrow\left\{\begin{array}{l}
c_{1,0}=\frac{1+d_{1,0} \lambda}{\lambda} \\
c_{1, n}=\frac{d_{1, n} \lambda \mathcal{K}_{0}\left(\frac{n \pi r}{\lambda}\right)+1}{\lambda \mathcal{I}_{0}\left(\frac{n \pi r}{\lambda}\right)} .
\end{array}\right.
\end{aligned}
$$

The condition on $\mathcal{G}$ as $r \rightarrow \infty$ (equation (46)) fixes $d_{1,0}=0$. Finally, $d_{1, n}$ can be determined using the jump condition on $\frac{\partial \mathcal{G}}{\partial r}$ (equation (45))

$$
\frac{d g_{n}}{d r}= \begin{cases}n \pi \frac{\left(d_{1, n} \lambda \mathcal{K}_{0}\left(\frac{n \pi \epsilon}{\lambda}\right)+1\right) \mathcal{I}_{1}\left(\frac{n \pi r}{\lambda}\right)}{\lambda^{2} \mathcal{I}_{0}\left(\frac{n \pi \epsilon}{\lambda}\right)} & \text { for } r<\epsilon, \\ -n \pi \frac{d_{1, n} \mathcal{K}_{1}\left(\frac{n \pi r}{\lambda}\right)}{\lambda} & \text { for } r>\epsilon\end{cases}
$$




$$
\begin{aligned}
\left.\frac{d g_{n}}{d r}\right|_{r=\epsilon^{-}} & -\left.\bar{\sigma} \frac{d g_{n}}{d r}\right|_{r=\epsilon^{+}}=0, \\
= & \frac{n \pi\left(d_{1, n} \lambda\left(\bar{\sigma} \mathcal{I}_{0}\left(\frac{n \pi \epsilon}{\lambda}\right) \mathcal{K}_{1}\left(\frac{n \pi \epsilon}{\lambda}\right)+\mathcal{I}_{1}\left(\frac{n \pi \epsilon}{\lambda}\right) \mathcal{K}_{0}\left(\frac{n \pi \epsilon}{\lambda}\right)\right)+\mathcal{I}_{1}\left(\frac{n \pi \epsilon}{\lambda}\right)\right)}{\lambda^{2} \mathcal{I}_{0}\left(\frac{n \pi \epsilon}{\lambda}\right)} \\
\Rightarrow & d_{1, n}=-\frac{\mathcal{I}_{1}\left(\frac{n \pi \epsilon}{\lambda}\right)}{\lambda\left(\bar{\sigma} \mathcal{I}_{0}\left(\frac{n \pi \epsilon}{\lambda}\right) \mathcal{K}_{1}\left(\frac{n \pi \epsilon}{\lambda}\right)+\mathcal{I}_{1}\left(\frac{n \pi \epsilon}{\lambda}\right) \mathcal{K}_{0}\left(\frac{n \pi \epsilon}{\lambda}\right)\right)}
\end{aligned}
$$

We now have the following explicit expression for $\mathcal{G}$, and can thus easily calculate $\frac{\partial \mathcal{G}}{\partial r}$, we have

$$
\begin{aligned}
& \mathcal{G}(X, r)=\left\{\begin{array}{ll}
\frac{1}{\lambda}+\sum_{n=1}^{\infty} \frac{\bar{\sigma} \mathcal{K}_{1}\left(\frac{n \pi \epsilon}{\lambda}\right)}{\lambda\left(\bar{\sigma} \mathcal{I}_{0}\left(\frac{n \pi \epsilon}{\lambda}\right) \mathcal{K}_{1}\left(\frac{n \pi \epsilon}{\lambda}\right)+\mathcal{I}_{1}\left(\frac{n \pi \epsilon}{\lambda}\right) \mathcal{K}_{0}\left(\frac{n \pi \epsilon}{\lambda}\right)\right)} \mathcal{I}_{0}\left(\frac{n \pi r}{\lambda}\right) \cos \left(\frac{n \pi X}{\lambda}\right) & \text { for } r<\epsilon \\
-\sum_{n=1}^{\infty} \frac{\mathcal{I}_{1}\left(\frac{n \pi \epsilon}{\lambda}\right)}{\lambda\left(\bar{\sigma} \mathcal{I}_{0}\left(\frac{n \pi \epsilon}{\lambda}\right) \mathcal{K}_{1}\left(\frac{n \pi \epsilon}{\lambda}\right)+\mathcal{I}_{1}\left(\frac{n \pi \epsilon}{\lambda}\right) \mathcal{K}_{0}\left(\frac{n \pi \epsilon}{\lambda}\right)\right)} \mathcal{K}_{0}\left(\frac{n \pi r}{\lambda}\right) \cos \left(\frac{n \pi X}{\lambda}\right) & \text { for } r>\epsilon
\end{array},\right. \\
& \frac{\partial \mathcal{G}}{\partial r}(X, r)= \begin{cases}\sum_{n=1}^{\infty} \frac{n \pi \bar{\sigma} \mathcal{K}_{1}\left(\frac{n \pi \epsilon}{\lambda}\right)}{\lambda^{2}\left(\bar{\sigma} \mathcal{I}_{0}\left(\frac{n \pi \epsilon}{\lambda}\right) \mathcal{K}_{1}\left(\frac{n \pi \epsilon}{\lambda}\right)+\mathcal{I}_{1}\left(\frac{n \pi \epsilon}{\lambda}\right) \mathcal{K}_{0}\left(\frac{n \pi \epsilon}{\lambda}\right)\right)} \mathcal{I}_{1}\left(\frac{n \pi r}{\lambda}\right) \cos \left(\frac{n \pi X}{\lambda}\right) & \text { for } r<\epsilon \\
\sum_{n=1}^{\infty} \frac{n \pi \mathcal{I}_{1}\left(\frac{n \pi \epsilon}{\lambda}\right)}{\lambda^{2}\left(\bar{\sigma} \mathcal{I}_{0}\left(\frac{n \pi \epsilon}{\lambda}\right) \mathcal{K}_{1}\left(\frac{n \pi \epsilon}{\lambda}\right)+\mathcal{I}_{1}\left(\frac{n \pi \epsilon}{\lambda}\right) \mathcal{K}_{0}\left(\frac{n \pi \epsilon}{\lambda}\right)\right)} \mathcal{K}_{1}\left(\frac{n \pi r}{\lambda}\right) \cos \left(\frac{n \pi X}{\lambda}\right) & \text { for } r>\epsilon\end{cases}
\end{aligned}
$$

The limit $r \rightarrow \epsilon$ is trivial, and can be taken simply by evaluating at $r=\epsilon$.

\section{Comparison to an analytic solution}

In this section, we consider a simplified case of equations (20)-(23) for a uniform cylindrical axon, giving the membrane a constant conductance (equivalent to an Ohmic leak current through the ion channels) instead of the complex, $\Phi$-dependent conductance described by the Hodgkin-Huxley model. 
This simplified model can be stated as follows

$$
\begin{aligned}
\nabla^{2} \phi & =0 \text { in } r<\epsilon \text { and } r>\epsilon, \\
\left.\frac{\partial \phi}{\partial r}\right|_{r=\epsilon^{-}} & =\left.\bar{\sigma} \frac{\partial \phi}{\partial r}\right|_{r=\epsilon^{+}}, \\
{[\phi]_{r=\epsilon^{+}}^{r=} } & =\Phi(x, t), \\
\mathcal{C} \frac{\partial \Phi}{\partial t} & =-\left.\frac{\partial \phi}{\partial r}\right|_{r=\epsilon^{-}}-g_{\text {leak }} \Phi(x, t),
\end{aligned}
$$

and

$$
\Phi(x, 0)=\cos (k x) .
$$

This has the solution

$$
\phi(x, r, t)=\left\{\begin{array}{ll}
\frac{\bar{\sigma} \mathcal{K}_{1}(k \epsilon)}{\bar{\sigma} \mathcal{I}_{0}(k \epsilon) \mathcal{K}_{1}(k \epsilon)+\mathcal{I}_{1}(k \epsilon) \mathcal{K}_{0}(k \epsilon)} \cos (k x) \mathcal{I}_{0}(k r) \times \\
\exp \left(-\left(\frac{k \bar{\sigma} \mathcal{I}_{1}(k \epsilon) \mathcal{K}_{1}(k \epsilon)}{C\left(\bar{\sigma} \mathcal{I}_{0}(k \epsilon) \mathcal{K}_{1}(k \epsilon)+\mathcal{I}_{1}(k \epsilon) \mathcal{K}_{0}(k \epsilon)\right)}+\frac{g_{\text {leak }}}{C}\right) t\right) & \text { for } r<\epsilon \\
-\frac{\bar{\sigma} \mathcal{I}_{1}(k \epsilon)}{\bar{\sigma} \mathcal{I}_{0}(k \epsilon) \mathcal{K}_{1}(k \epsilon)+\mathcal{I}_{1}(k \epsilon) \mathcal{K}_{0}(k \epsilon)} \cos (k x) \mathcal{K}_{0}(k r) \times & \\
\exp \left(-\left(\frac{k \bar{\sigma} \mathcal{I}_{1}(k \epsilon) \mathcal{K}_{1}(k \epsilon)}{C\left(\bar{\sigma} \mathcal{I}_{0}(k \epsilon) \mathcal{K}_{1}(k \epsilon)+\mathcal{I}_{1}(k \epsilon) \mathcal{K}_{0}(k \epsilon)\right)}+\frac{g_{\text {leak }}}{C}\right) t\right) & \text { for } r>\epsilon
\end{array},\right.
$$

which yields the following expression for the transmembrane potential

$\Phi(x, t)=\cos (k x) \exp \left(-\left(\frac{k \bar{\sigma} \mathcal{I}_{1}(k \epsilon) \mathcal{K}_{1}(k \epsilon)}{C\left(\bar{\sigma} \mathcal{I}_{0}(k \epsilon) \mathcal{K}_{1}(k \epsilon)+\mathcal{I}_{1}(k \epsilon) \mathcal{K}_{0}(k \epsilon)\right)}+\frac{g_{\text {leak }}}{C}\right) t\right)$,

where $\mathcal{I}_{\alpha}(x)$ and $\mathcal{K}_{\alpha}(x)$ are the modified Bessel functions of the first and second kind respectively.

We verify the accuracy of our numerical scheme by using it to solve equations (87)-(90), and note that the method is robust with respect to changes in resolution and period, $\lambda$. Figure 11 shows this comparison graphically, and figure 12 shows how the errors can be reduced to the scale of machine accuracy by taking sufficiently many time steps. 


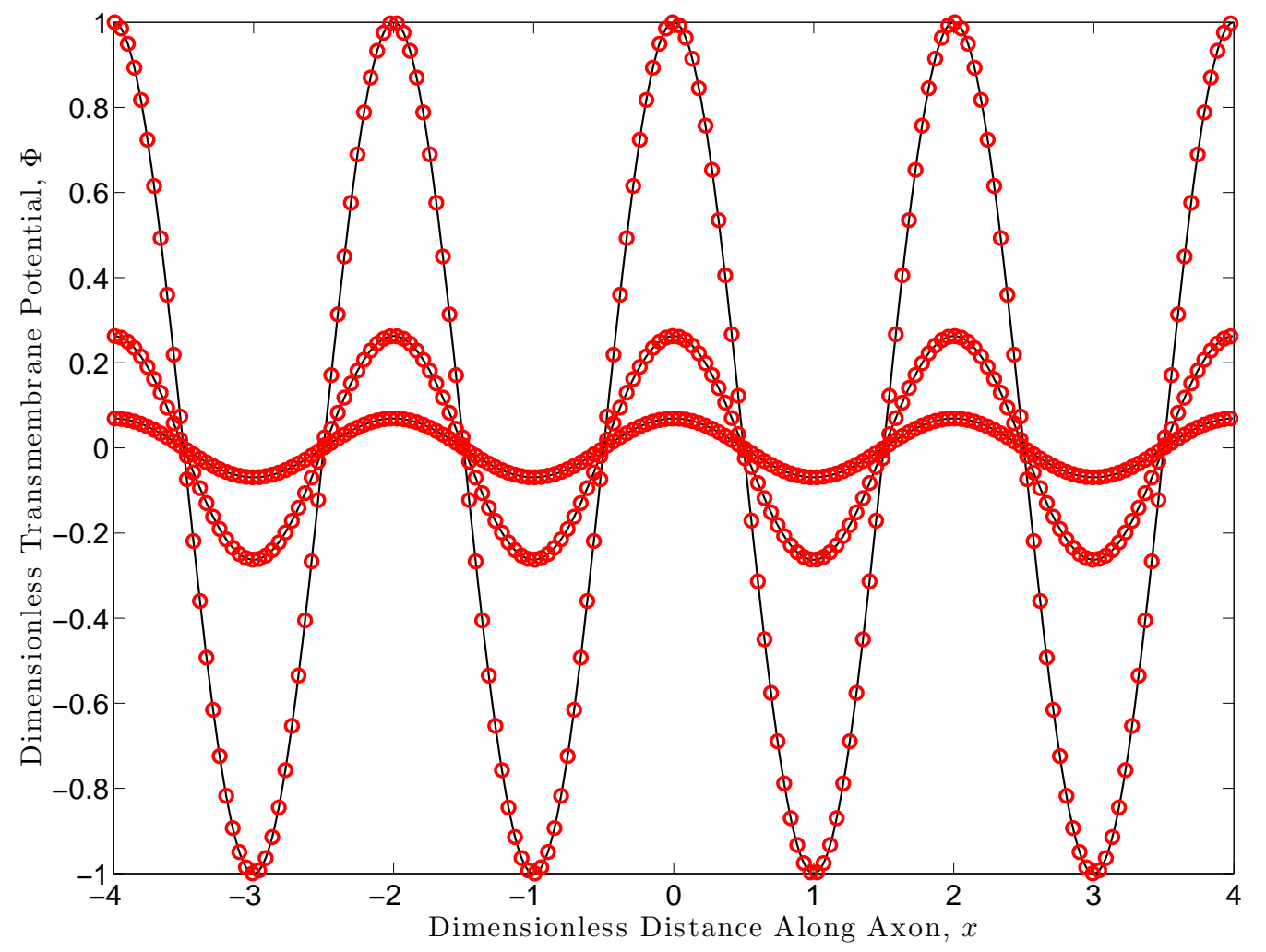

Figure 11: Numerical (solid curve) and analytic (circles) solutions to equations (87)-(90), at times $t=0,0.025,0.05$, using 512 space points and 5000 time steps 


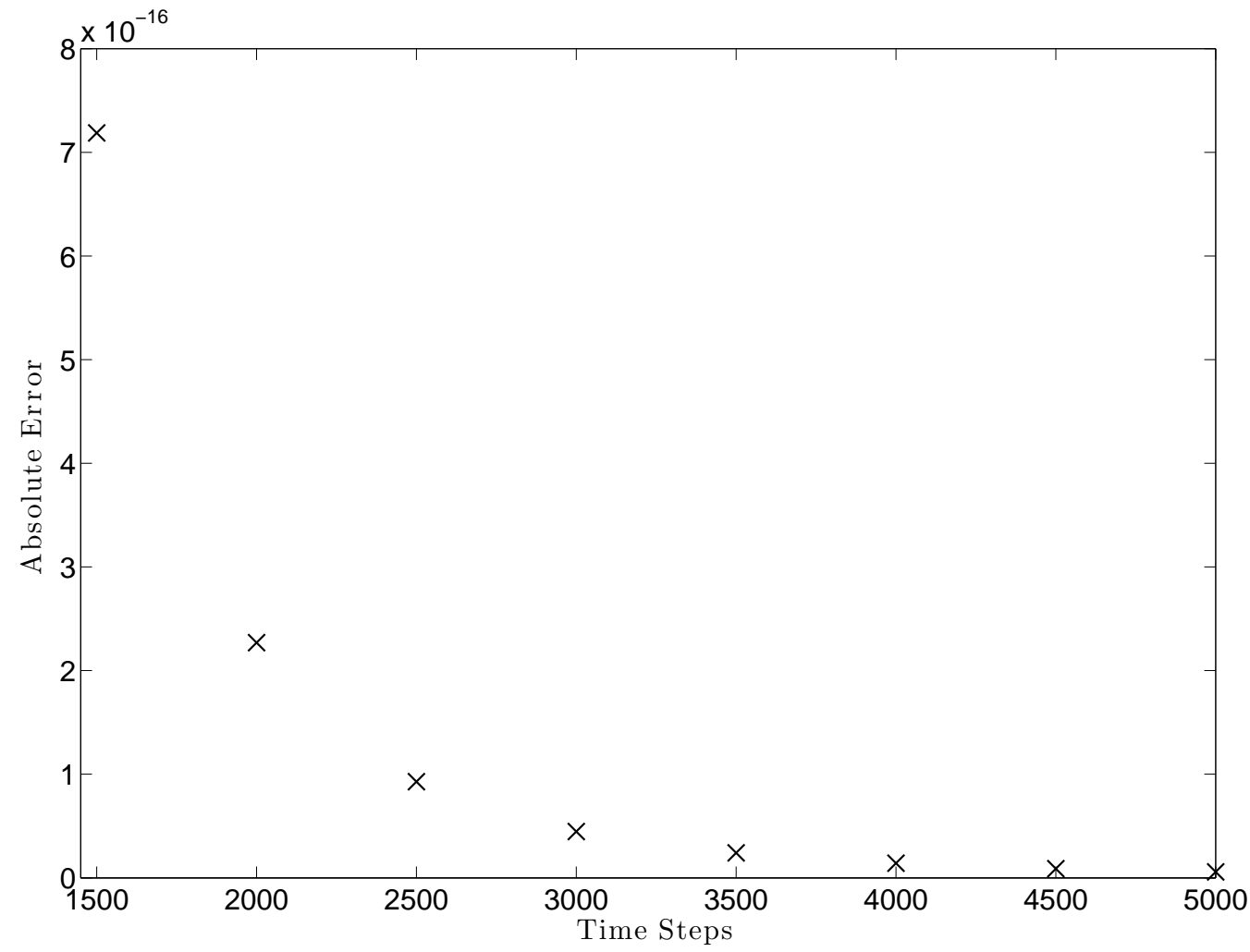

Figure 12: Mean absolute error in simulation at $t=0.25$ as a function of number of time steps, using 512 space steps 


\section{References}

RK Adair. Optimum ion channel properties in the squid giant axon. Phys Rev E, 69(4):042902, 2004.

KW Altman and R Plonsey. Point source nerve bundle stimulation: Effects of fiber diameter and depth on simulated excitation. IEEE Trans Biomed Eng, 37(7):688-698, 1990.

CM Armstrong and F Benzanilla. Inactivation of the sodium channel: Ii. gating current experiments. J Gen Physiol, 70:567-590, 1977.

BP Bean. Sodium channel inactivation in the crayfish giant axon. Biophys $J, 35: 595-614,1981$.

RS Bear, FO Schmitt, and JZ Young. The sheath components of the giant nerve fibres of the squid. P Roy Soc Lond B Bio, 123(833):496-504, 1937.

F Benzanilla and CM Armstrong. Inactivation of the sodium channel: I. sodium current experiments. J Gen Physiol, 70:549-566, 1977.

AA Faisal and SB Laughlin. Stochastic simulations on the reliability of action potential propagation in thin axons. PLoS Comput Biol, 3(5):783-795, May 2007.

W Gerstner and WM Kistler. Spiking Neuron Models: Single Neurons, Populations, Plasticity. Cambridge University Press, August 2002.

B Hille. Ionic Channels of Excitable Membranes. Sinauer Associates, 3 edition, 2001.

AL Hodgkin. A note on conduction velocity. J Physiol, 125(221-224), 1954.

AL Hodgkin and AF Huxley. A quantitative description of membrane current and its application to conduction and excitation in nerve. $J$ Physiol, 117: 500-544, 1952a.

AL Hodgkin and AF Huxley. The components of membrane conductance in the giant axon of loligo. J Physiol, 116:473-496, 1952b.

AL Hodgkin and AF Huxley. Currents carried by sodium and potassium ions through the membrane of the giant axon of loligo. J Physiol, 116:449-472, 1952c. 
AL Hodgkin and AF Huxley. The dual effect of membrane potential on sodium conductance in the giant axon of loligo. J Physiol, 116:497-506, 1952d.

AL Hodgkin and AF Huxley. Propagation of electrical signals along giant nerve fibres. P Roy Soc Lond B Bio, 140(899):177-183, 1952e.

AL Hodgkin, AF Huxley, and B Katz. Measurement of current-voltage relations in the membrane of the giant axon of loligo. J Physiol, 116:424-448, 1952.

GM Hughes and L Tauc. An electrophysiological study of the anatomical relations of two giant nerve cells in Aplysia Depilans. J Exp Biol, 40: 469-486, 1963.

B Katz and R Miledi. The characteristics of 'end-plate noise' produced by different depolarizing drugs. J Physiol, 230:707-717, 1973.

W Krassowska and JC Neu. Homogenization of syncytial tissues. Crit Rev Biomed Eng, 21:137-199, 1993.

KA Lindsay, JR Rosenberg, and G Tucker. From maxwell's equation to the cable equation and beyond. Prog Biophys Mol Bio, 85:71-116, 2004.

I Llano, CK Webb, and F Bezanilla. Potassium conductance of the squid giant axon: Single channel studies. J Gen Physiol, 22:179-196, 1988.

SS Nagarajan and DM Durand. Analysis of magnetic stimulation of a concentric axon in a nerve bundle. IEEE Trans Biomed Eng, 42(9), 1995.

E Neher and B Sakmann. Single-channel currents recorded from membrane of denervated frog muscle fibres. Nature, 260:799-802, 1976.

E Neher, B Sakmann, and JH Steinbach. The extracellular patch clamp: A method for resolving currents through individual open channels in biological membranes. Pflug Arch Eur J Phy, 375(2):219-228, 1978.

TS Otis and WF Gilly. Jet-propelled escape in the squid loligo opalescens: concerted control by giant and non-giant motor axon pathways. P Natl Acad Sci USA, 87(8):2911-2915, 1990. 
JW Perram and PJ Stiles. A dynamical system for action potentials in the giant axon of the squid. J Phys Chem-US, 114:20350-20361, 2010.

T Preuss and WF Gilly. Role of prey-capture experience in the development of the escape response in the squid loligo opalescens: A physiological correlate in an identified neuron. $J$ Exp Biol, 203:559-565, 2000.

D Purves, GJ Augustine, D Fitzpatrick, WC Hall, A LaMantia, and LE White, editors. Neuroscience. Sinauer Associates, 5 edition, 2011.

F Rattay. Analysis of models for external stimulation of axons. IEEE Trans Biomed Eng, 33(10):974-977, 1986.

G Richardson. A multiscale approach to modelling electrochemical processes occurring across the cell membrane with application to transmission of action potentials. Math Med Biol, 26(3):201-224, 2009.

G Richardson and SJ Chapman SJ. Derivation of the bidomain equations for a beating heart with a general microstructure. SIAM J Appl Math, 71 (3):657-675, 2011.

R Stampfli. Bau und funktion isolierter markhaltiger nervenfasern. Ergeb Physiol, 47:70, 1952.

AF Strassberg and LJ DeFelice. Limitations of the hodgkin-huxley formalism: Effects of single channel kinetics on transmembrane voltage dynamics. Neural Comput, 5:843-855, 1993.

W Thompson. On the theory of the electric telegraph. Proc $R$ Soc, 1855.

CA Vandenberg and F Benzanilla. A sodium channel gating model based on single channel, macroscopic ionic, and gating currents in the squid axon. Biophys J, 60(6):1511-1533, 1991. 